\title{
Complications of cranioplasty in relationship to traumatic brain injury: a systematic review and meta-analysis
}

\author{
David Shepetovsky $^{1}$ (D) $\cdot$ Gianluca Mezzini $^{1} \cdot$ Lorenzo Magrassi $^{1,2}$ (D) \\ Received: 17 August 2020 / Revised: 18 February 2021 / Accepted: 22 February 2021 / Published online: 8 March 2021 \\ (C) The Author(s) 2021
}

\begin{abstract}
Despite being a common procedure, cranioplasty (CP) is associated with a variety of serious, at times lethal, complications. This study explored the relationship between the initial injury leading to decompressive craniectomy (DC) and the rates and types of complications after subsequent CP. It specifically compared between traumatic brain injury (TBI) patients and patients undergoing $\mathrm{CP}$ after $\mathrm{DC}$ for other indications.

A comprehensive search of PubMed, Scopus, and the Cochrane Library databases using PRISMA guidelines was performed to include case-control studies, cohorts, and clinical trials reporting complication data for CP after DC. Information about the patients' characteristics and the rates of overall and specific complications in TBI and non-TBI patients was extracted, summarized, and analyzed.

A total of 59 studies, including the authors' institutional experience, encompassing 9264 patients (4671 TBI vs. 4593 nonTBI) met the inclusion criteria; this total also included 149 cases from our institutional series. The results of the analysis of the published series are shown both with and without our series 23 studies reported overall complications, 40 reported infections, 10 reported new-onset seizures, 13 reported bone flap resorption (BFR), 5 reported post-CP hydrocephalus, 10 reported intracranial hemorrhage (ICH), and 8 reported extra-axial fluid collections (EFC). TBI was associated with increased odds of BFR (odds ratio $[\mathrm{OR}] 1.76, p<0.01)$ and infection (OR 1.38, $p=0.02$ ). No difference was detected in the odds of overall complications, seizures, hydrocephalus, ICH, or EFC.

Awareness of increased risks of BFR and infection after CP in TBI patients promotes the implementation of new strategies to prevent these complications especially in this category of patients.
\end{abstract}

Keywords Cranioplasty $\cdot$ Decompressive craniectomy $\cdot$ Traumatic brain injury $\cdot$ Complications

\section{Introduction}

Management of severe intracranial hypertension, when conservative therapies fail, may require DC, which is often followed by $\mathrm{CP}$ after patient stabilization and optimization $[1,2]$. Despite being a time-honored and seemingly

Lorenzo Magrassi

lorenzo.magrassi@unipv.it

David Shepetovsky

shep.david@gmail.com

Gianluca Mezzini

gianluca.mezzini@yahoo.it

1 Department of Clinical Surgical Diagnostic and Pediatric Sciences, University of Pavia, Viale Brambilla 74, 27100 Pavia, Italy

2 IRCCS Fondazione Policlinico S. Matteo, Pavia, Italy "straightforward" procedure, CP is burdened by a complication rate of up to $40 \%$, some of which are severe enough to compromise the outcome of the initial brain injury [3-5]. Documented complications following $\mathrm{CP}$ include, among others: seizures, ICH, EFC, infections, hydrocephalus, BFR, mechanical complications (MC), cerebral edema, and neurological deficits (NDs) [3, 6-9].

In recent years, a considerable number of studies explored the various risk factors and pathological mechanisms underlying complications of $\mathrm{CP}$ after different brain injuries not limited to trauma. However, very few of those studies evaluated whether the type of initial injury may represent a risk factor for post-CP complications [10-12].

Victims of TBI constitute the largest group of patients requiring DC and subsequent CP [13]. In those patients, factors such as impaired wound sterility due to traumatic contamination, scalp lacerations, multiple complex skull fractures, 
diffuse axonal injury, and pericontusional ischemia may all increase the risk of post-CP complications and be detrimental to the long-term outcome of the patient $[2,14-16]$.

This study aimed to compare the rates and types of post-CP complications between TBI patients and patients suffering from other primary pathologies, via a systematic review and meta-analysis of the literature, supplemented by our institutional experience. By elucidating the relationship between TBI and specific complications following CP, it may be possible to prevent neurological damage and minimize the risk of complications linked to $\mathrm{CP}$, thus potentially improving neurologic outcome.

\section{Materials and methods}

\section{Search strategy}

A comprehensive literature search to identify articles of interest was performed in accordance with the PRISMA (Preferred Reporting Items for Systematic Reviews and Meta-Analyses) guidelines [17]. PubMed, Scopus, and the Cochrane Library were searched using the keywords: "cranioplasty," "decompressive craniectomy," "complication," "outcome," "traumatic brain injury," and "TBI." The search included all papers published between January 2000 and May 2020. The reference sections of the identified studies were reviewed to identify any additional relevant articles.

\section{Study selection}

Case-control studies, cohort studies, or clinical trials reporting on the relationship between the indication for DC and the type and rate of related post-CP complications in human adults were included in the analyses, unless they did not include enough data to calculate the number of patients that underwent DC for each indication considered in the study or the number of complications following subsequent $\mathrm{CP}$.

Comments, letters, technical notes, editorials, case reports, or case series relating individually selected cases were excluded. Meta-analyses and reviews were also excluded; however, referenced articles were thoroughly screened for possible inclusion.

Non-English articles were excluded, unless the article was part of a related systematic review. Studies that involved animals, included non-calvarial or maxillofacial procedures, or focused exclusively on the pediatric population were excluded. Studies were excluded if they reported patients who initially underwent craniotomy for reasons other than decompression unless it was possible to separate them from the DC patients.

Table 1 summarizes the inclusion and exclusion criteria.

\section{Data extraction}

The following information was collected from eligible articles: (1) number of patients in the cohort, (2) indication for DC, (3) mean age, (4) male to female ratio, (5) time interval between DC and CP, (6) implant materials used for CP (e.g., preserved autologous bone, methyl methacrylate, and polyether ether ketone), (7) mean post-op follow-up time, and (8) incidence and types of post-CP complications.

Complications were grouped into the following categories: (1) new-onset seizures; (2) intracranial hemorrhage (including intracerebral hemorrhage, subdural hematoma, and epidural hematoma); (3) nonhemorrhagic extra-axial fluid collections (including subgaleal and subdural effusion, cerebrospinal fluid leak or fistula, and hygroma); (4) infection (including surgical site infection, wound healing disturbances and wound dehiscence, graft infection, empyema, brain abscess, and osteomyelitis); (5) hydrocephalus (treated with VPS or conservatively); (6) bone flap resorption (including aseptic bone flap necrosis; diagnosed by clinical exam (softness of the reimplanted flap at palpation and/or flap loosening), imaging (CT scan), or both). In our own series, bone flap resorption was defined as any bone defect superior to $0.5 \mathrm{~cm}$ in its largest diameter when a late CT scan (obtained at least 2 months after $\mathrm{CP}$ ) was compared to postoperative (within $24 \mathrm{~h}$ from CP) CT scan; (7) other complications include MC, cerebral edema, and NDs. For studies that reported all cases of complications observed in their cohort (rather than focusing only on specific types of complications), the category "overall complications" was calculated by including all subjects affected by one or more of the above complications.

Not all articles provided information about each item; therefore, comparative analysis was limited by the nature of the source data.

Study quality and risk of bias of individual articles were assessed by the Newcastle-Ottawa Scale (NOS) - a three-category, 9-point scale assessing cohort selection, comparability, and outcome [18]. A higher score indicates higher quality.

\section{Local series of CP patients}

A retrospective review was done to identify all cranioplasties performed on adult patients (older than eighteen at the time of the initial injury) at the neurosurgical unit of Fondazione IRCCS San Matteo (Pavia, Italy), between January 1st 2009 and December 31st 2019. All patients or their legal representative when the patient neurological status did not allow an informed decision signed an informed consent authorizing the use of anonymized clinical data for retrospective analysis and clinical research. Relevant clinical and demographic data were extracted from the patients' medical records, including age, sex, indication for initial DC, timing of CP after initial DC, CP implant material, postoperative complications, and post-CP 
Table 1 Study selection criteria

\begin{tabular}{ll}
\hline Inclusion criteria & Exclusion criteria \\
\hline $\begin{array}{l}\text { Case-control studies, cohort studies, or clinical trials, } \\
\text { large case series }\end{array}$ & $\begin{array}{c}\text { Comments, letters, technical notes, editorials, } \\
\text { meta-analyses, reviews, case reports, or individually } \\
\text { selected case series }\end{array}$ \\
$\begin{array}{l}\text { Includes data on the indication for decompressive } \\
\text { craniectomy and the type and rate of related } \\
\text { post-cranioplasty complications }\end{array}$ & $\begin{array}{c}\text { Focuses on animals, pediatric patients, or } \\
\text { cranioplasties done for reasons other than } \\
\text { post-decompressive craniectomy } \\
\text { Published in a language other than English } \\
\text { Contains insufficient data for meta-analysis }\end{array}$ \\
\hline
\end{tabular}

follow-up time. All data were anonymized before analysis, and parameters characterizing our series were introduced as a bulk in the meta-analysis.

\section{Data analysis}

The data were analyzed using Review Manager 5.3.5 (The Cochrane Collaboration) [19]. Complications were first categorized by type (e.g., overall complications, infection, and seizure) and then split into two groups: "TBI patients" and "non-TBI patients," according to the original indication for initial DC. TBI patients included every injury described as "trauma" or "head injury" as well as concussion, contusion, and traumatic/non-spontaneous ICH. Open and closed TBI were considered together. The non-TBI patient group included all other indications such as hemorrhagic and ischemic strokes, spontaneous subarachnoid hemorrhage, infections, and tumors.

The pooled rate of every complication type for each indication group was calculated by summing the number of patients reported to suffer the specific complication in each study and then dividing the by the total number of patients who underwent DC for that indication.

For studies that included both TBI and non-TBI patients, odds ratios (ORs) and 95\% confidence intervals (CI) for each complication were calculated by dividing the odds in the "TBI" patient group by the odds in the "non-TBI" patient group. To account for the heterogeneity of the data, the Mantel-Haenszel method with random-effects model was used. The $I^{2}$ metric was reported to further quantify heterogeneity. $p$ values of 0.05 or less were considered statistically significant.

\section{Results}

The initial literature search yielded 738 entries. Eighteen additional articles were found by searching the references of articles. Two-hundred-thirty-four duplicates were found and removed. The remaining 522 studies were screened, and 457 were eliminated based on exclusion criteria. After this, 65 articles were assessed for eligibility and 59 were included in the final analysis (Fig. 1) [10-12, 15, 16, 20-72].

Six articles were excluded for the following reasons: 5 articles utilized the same 2 cohorts [10, 37, 73-75]. Therefore, 1 article for each cohort was selected and the other 3 articles were discarded. One article dealt mostly with pediatric patients (mean age 12.2 years) [76]; 2 articles included a significant proportion $(>75 \%)$ of cranioplasties done for reasons other than post-DC $[77,78]$.

Two studies included cranioplasties done both following a DC and for other indications but reported sufficient detail to allow for extraction of the data regarding post-DC cranioplasties only [45, 49].

There was an overlap between 2 studies in the authors, location and time frame in which they were performed [16, 36]. However, the studies focused on different complications (1 reported BFR, and the other dealt with infection, EFC, and $\mathrm{IH}$ ), and were therefore treated as a single cohort with complication rates pooled from both studies.

Seven of the included studies were published between 2000 and 2009, compared with 52 studies published between 2010 and 2019. This disparity reflects the growing interest for complications of CP as demonstrated by PubMed's Publication Timeline feature showing an average of $3 \pm 3$ papers per year during the first decade of the century compared with $35 \pm 13$ per year during the second decade.

\section{Study and patient characteristics}

The baseline characteristics of the individual studies are summarized in Table 2. Of the studies included, 6 were prospective cohorts and the rest $(n=53)$ were retrospective cohorts. Together, the studies comprised a pooled cohort of 9264 patients, out of which 4671 (50\%) were affected by TBI. Fifty studies reported mean age, with a pooled mean of $44 \pm 1$ years. Fifty-six studies reported data about the male to female ratio, with a pooled mean of $65 \% \pm 2 \%$ males.

Fifty-five studies included CP performed using autologous bone flaps $(\mathrm{ABF})$, with 36 studies also including synthetic materials such as titanium mesh, hydroxyapatite (HA), polymethyl methacrylate (PMMA), polyether-ether-ketone 
Fig. 1 PRISMA flow diagram of literature search strategy and article selection

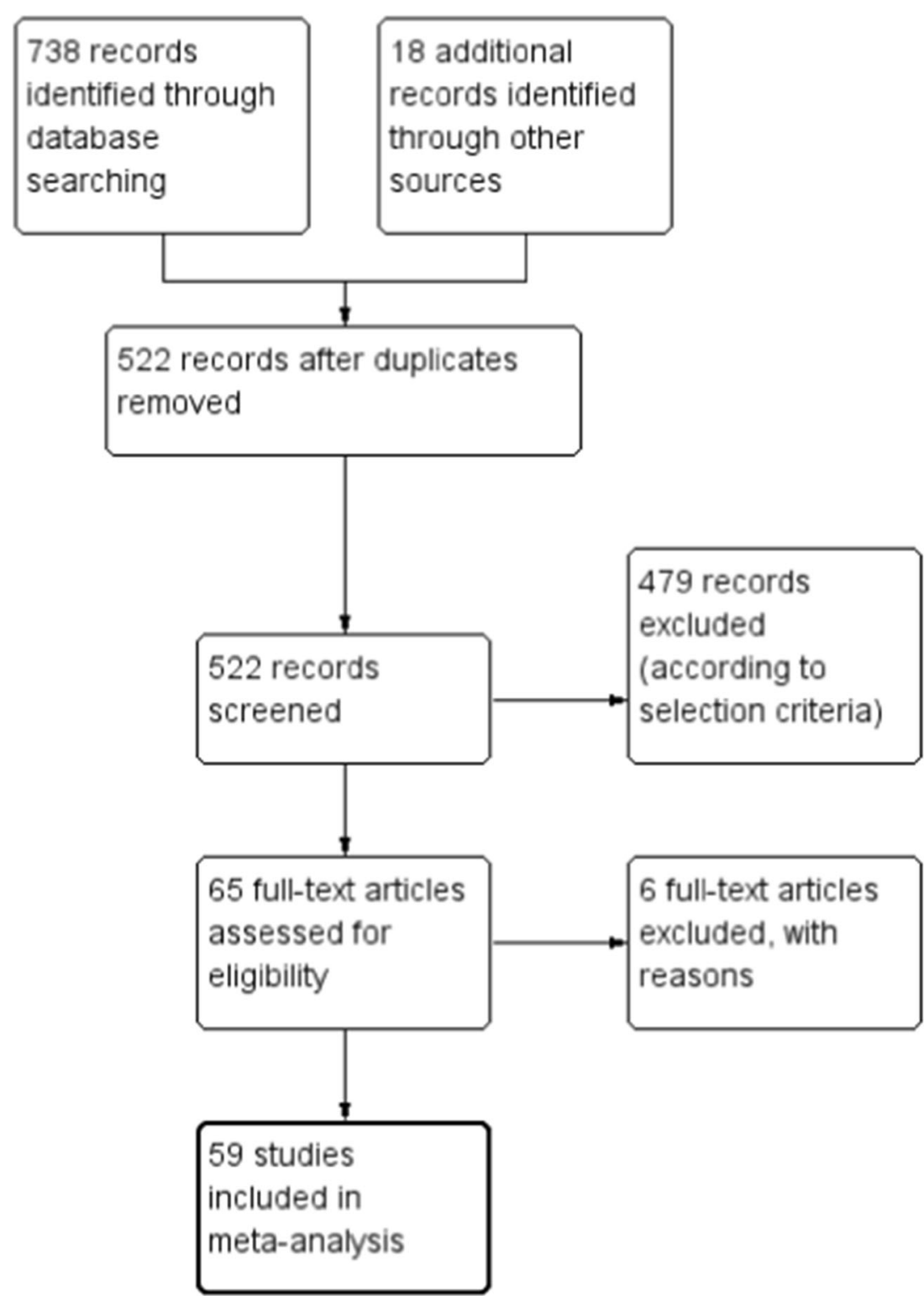

(PEEK), and individually customized implants (containing a combination of the previously mentioned materials). Three studies included only CP performed with synthetic materials.

Time interval from DC to CP ranged between 39 and 370 days, with a median of 196 and a mean of $161 \pm 37.5$ days. Fourteen studies, including our own series, stratified the time interval according to the initial indication for DC $[16,22,24$, $25,29,30,36,37,45,53,55,56,67]$. The pooled mean interval from those studies was $131 \pm 62$ days for TBI patients, compared with $116 \pm 58$ days for non-TBI patients. The timing of CP did not significantly differ between the underlying pathologies $($ difference $=15$ days, $95 \% \mathrm{CI}=-37<\operatorname{diff}<$ 67 , and $p=0.57$ ).

Study quality ranged from 5 to 8 out of 9 on the NewcastleOttawa Scale. Points were deduced mainly for lack of matched cohorts, neglect to mention length of follow-up and lack of representativeness of the exposed cohort of the overall population. Most $(n=56)$ had adequate ascertainment of exposure, sufficient comparability, and good assessment of outcome.

\section{Institutional experience}

The general characteristics of our cohort are summarized in Table 2. It included a total of 149 patients, out of which 56 (37.6\%) were initially diagnosed with TBI. The overall complication rate of CP was calculated by considering all complications independently of whether they required treatment or not and added up to $42.3 \%$. There was no statistically significant difference in odds of overall complications between TBI patients $(37.5 \%)$ and non-TBI patients (45.2\%); OR $=0.83$ ( $p=0.56$ and CI 0.45-1.54). 
Table 2 Characteristics of included studies

\begin{tabular}{|c|c|c|c|c|c|c|c|c|c|c|c|c|}
\hline \multirow[t]{2}{*}{ Reference } & \multirow[t]{2}{*}{ Study design } & \multicolumn{3}{|c|}{$\begin{array}{l}\text { Number of } \\
\text { patients }\end{array}$} & \multirow{2}{*}{$\begin{array}{l}\text { Mean } \\
\text { age } \\
\text { (years) }\end{array}$} & \multirow[t]{2}{*}{$\begin{array}{l}\text { Males } \\
(\%)\end{array}$} & \multirow[t]{2}{*}{$\begin{array}{l}\text { Indications } \\
\text { for DC }\end{array}$} & \multirow[t]{2}{*}{$\begin{array}{l}\text { Described } \\
\text { complications }\end{array}$} & \multirow[t]{2}{*}{$\begin{array}{l}\text { Implant } \\
\text { material }\end{array}$} & \multirow{2}{*}{$\begin{array}{l}\text { Mean } \\
\text { DC to } \\
\text { CP } \\
\text { interval } \\
\text { (days) }\end{array}$} & \multirow{2}{*}{$\begin{array}{l}\text { Follow- } \\
\text { up } \\
\text { (months) }\end{array}$} & \multirow[t]{2}{*}{$\begin{array}{l}\text { Quality } \\
\text { (NOS) }\end{array}$} \\
\hline & & Total & TBI & $\begin{array}{l}\text { Non- } \\
\text { TBI }\end{array}$ & & & & & & & & \\
\hline Nagayama $2002 *$ & $\begin{array}{l}\text { Retrospective } \\
\text { cohort }\end{array}$ & 195 & 28 & 167 & NS & NS & $\begin{array}{l}\text { TBI, } \\
\text { non-TBI }\end{array}$ & Infection & $\mathrm{ABF}$ & NS & NS & N/A \\
\hline Shimizu $2002 *$ & $\begin{array}{l}\text { Retrospective } \\
\text { cohort }\end{array}$ & 70 & 29 & 41 & NS & NS & $\begin{array}{l}\text { TBI, } \\
\text { non-TBI }\end{array}$ & Infection & $\mathrm{ABF}$ & NS & NS & N/A \\
\hline Iwama 2003 & $\begin{array}{l}\text { Retrospective } \\
\text { cohort }\end{array}$ & 49 & 15 & 34 & 51 & 61 & $\begin{array}{c}\text { SAH, TBI, } \\
\text { VL, ICH, } \\
\text { stroke, } \\
\text { tumor, } \\
\text { infection }\end{array}$ & $\begin{array}{l}\text { Infection, } \\
\text { BFR }\end{array}$ & $\mathrm{ABF}$ & NS & 59 & $6 \star$ \\
\hline $\begin{array}{l}\text { Moreira-Gonzalez } \\
2003\end{array}$ & $\begin{array}{l}\text { Retrospective } \\
\text { cohort }\end{array}$ & 200 & 99 & 101 & 32 & 55 & TBI, tumor & NS & $\begin{array}{l}\text { ABF, PMMA, } \\
\text { HA }\end{array}$ & NS & 36 & $7 \star$ \\
\hline Matsuno 2006 & $\begin{array}{l}\text { Retrospective } \\
\text { cohort }\end{array}$ & 206 & 64 & 142 & 48 & 60 & $\begin{array}{c}\text { SAH, ICH, } \\
\text { VL, TBI, } \\
\text { infection, } \\
\text { tumor }\end{array}$ & Infection & $\begin{array}{r}\text { ABF, PMMA, } \\
\text { customized }\end{array}$ & NS & 64 & $7 \star$ \\
\hline Kriegel 2007 & $\begin{array}{l}\text { Retrospective } \\
\text { cohort }\end{array}$ & 39 & 11 & 28 & 40 & 56 & $\begin{array}{l}\text { TBI, stroke, } \\
\text { ICH, } \\
\text { tumor, } \\
\text { infection }\end{array}$ & $\begin{array}{l}\text { Infection, } \\
\text { BFR, ICH }\end{array}$ & ABF, PMMA & 234 & 32 & $6 \star$ \\
\hline Cheng 2008 & $\begin{array}{l}\text { Retrospective } \\
\text { cohort }\end{array}$ & 84 & 60 & 24 & 45 & 73 & $\begin{array}{l}\text { TBI, ICH, } \\
\text { stroke, } \\
\text { ST, VL, } \\
\text { tumor, } \\
\text { infection }\end{array}$ & Infection & ABF, PMMA & 370 & NS & $6 \star$ \\
\hline Beauchamp 2010 & $\begin{array}{l}\text { Retrospective } \\
\text { cohort }\end{array}$ & 69 & 69 & 0 & $30^{\wedge}$ & 80 & TBI & $\mathrm{HC}$, infection & $\begin{array}{l}\mathrm{ABF}, \\
\text { synthetic }\end{array}$ & $87^{\wedge}$ & NS & $6 \star$ \\
\hline Chang 2010 & $\begin{array}{l}\text { Retrospective } \\
\text { cohort }\end{array}$ & 212 & 79 & 133 & 44 & 57 & $\begin{array}{l}\text { SAH, TBI, } \\
\text { stroke, } \\
\text { tumor, } \\
\text { infection, } \\
\text { VL, other }\end{array}$ & NS & $\begin{array}{l}\mathrm{ABF}, \\
\text { synthetic }\end{array}$ & 160 & NS & $7 \star$ \\
\hline Inamasu 2010 & $\begin{array}{l}\text { Retrospective } \\
\text { cohort }\end{array}$ & 70 & 33 & 37 & 46 & 53 & $\begin{array}{c}\text { TBI, SAH, } \\
\text { ICH, } \\
\text { stroke }\end{array}$ & Infection & $\mathrm{ABF}$ & 39 & 50 & $7 \star$ \\
\hline Stephens 2010 & $\begin{array}{l}\text { Retrospective } \\
\text { cohort }\end{array}$ & 108 & 108 & 0 & 26 & 99 & TBI & $\begin{array}{l}\text { Infection, } \\
\text { seizure, } \\
\text { ICH }\end{array}$ & PMMA, TM & 190 & NS & $6 \star$ \\
\hline Güresir 2011 & $\begin{array}{l}\text { Prospective } \\
\text { cohort }\end{array}$ & 196 & 74 & 122 & 51 & 55 & $\begin{array}{l}\text { TBI, SAH, } \\
\text { ICH, } \\
\text { stroke, } \\
\text { other }\end{array}$ & $\begin{array}{l}\text { Infection, } \\
\text { EFC, ICH }\end{array}$ & $\mathrm{ABF}$ & 97 & $>6$ & $8 \star$ \\
\hline Huang 2011 & $\begin{array}{l}\text { Prospective } \\
\text { cohort }\end{array}$ & 135 & 135 & 0 & 43 & 69 & TBI & $\begin{array}{l}\text { Infection, } \\
\text { ICH, EFC }\end{array}$ & $\mathrm{ABF}$ & 83 & 33 & $6 \star$ \\
\hline Lee 2011 & $\begin{array}{l}\text { Retrospective } \\
\text { cohort }\end{array}$ & 59 & 39 & 20 & 48 & 66 & $\begin{array}{l}\text { TBI, SAH, } \\
\text { stroke, } \\
\text { tumor }\end{array}$ & $\begin{array}{l}\mathrm{EFC}, \mathrm{ICH}, \\
\text { infection, } \\
\mathrm{BFR}\end{array}$ & ABF, PMMA & 196 & 11.5 & $8 \star$ \\
\hline Sobani 2011 & $\begin{array}{l}\text { Retrospective } \\
\text { cohort }\end{array}$ & 96 & 55 & 41 & 33 & 73 & $\begin{array}{l}\text { TBI, tumor, } \\
\text { infection, } \\
\text { stroke }\end{array}$ & $\begin{array}{l}\mathrm{HC}, \\
\text { infection, } \\
\mathrm{ICH}, \\
\text { seizures, } \\
\text { EFC, other }\end{array}$ & $\begin{array}{r}\text { ABF, PMMA, } \\
\text { customized }\end{array}$ & 90 & 13 & $8 \star$ \\
\hline Archavalis 2012 & $\begin{array}{l}\text { Retrospective } \\
\text { cohort }\end{array}$ & 200 & 51 & 149 & $53^{\wedge}$ & 55 & $\begin{array}{l}\text { SAH, TBI, } \\
\text { stroke, } \\
\text { ICH, } \\
\text { infection }\end{array}$ & $\begin{array}{l}\text { Infection, } \\
\text { ICH, EFC, } \\
\text { other }\end{array}$ & $\mathrm{ABF}$ & 75 & 43 & $8 \star$ \\
\hline $\operatorname{Im} 2012$ & $\begin{array}{l}\text { Retrospective } \\
\text { cohort }\end{array}$ & 131 & 61 & 70 & 50 & 62 & $\begin{array}{l}\text { VL, TBI, } \\
\text { tumor }\end{array}$ & Infection & $\begin{array}{l}\text { ABF, PMMA, } \\
\text { PEEK, }\end{array}$ & $<90$ & NS & $6 \star$ \\
\hline
\end{tabular}


Table 2 (continued)

\begin{tabular}{|c|c|c|c|c|c|c|c|c|c|c|c|c|}
\hline \multirow[t]{2}{*}{ Reference } & \multirow[t]{2}{*}{ Study design } & \multicolumn{3}{|c|}{$\begin{array}{l}\text { Number of } \\
\text { patients }\end{array}$} & \multirow{2}{*}{$\begin{array}{l}\text { Mean } \\
\text { age } \\
\text { (years) }\end{array}$} & \multirow[t]{2}{*}{$\begin{array}{l}\text { Males } \\
(\%)\end{array}$} & \multirow[t]{2}{*}{$\begin{array}{l}\text { Indications } \\
\text { for DC }\end{array}$} & \multirow[t]{2}{*}{$\begin{array}{l}\text { Described } \\
\text { complications }\end{array}$} & \multirow[t]{2}{*}{$\begin{array}{l}\text { Implant } \\
\text { material }\end{array}$} & \multirow{2}{*}{$\begin{array}{l}\text { Mean } \\
\text { DC to } \\
\text { CP } \\
\text { interval } \\
\text { (days) }\end{array}$} & \multirow{2}{*}{$\begin{array}{l}\text { Follow- } \\
\text { up } \\
\text { (months) }\end{array}$} & \multirow[t]{2}{*}{$\begin{array}{l}\text { Quality } \\
\text { (NOS) }\end{array}$} \\
\hline & & Total & TBI & $\begin{array}{l}\text { Non- } \\
\text { TBI }\end{array}$ & & & & & & & & \\
\hline & & & & & & & & & $\begin{array}{l}\text { bone } \\
\text { cement }\end{array}$ & & & \\
\hline Schuss 2012 & $\begin{array}{l}\text { Prospective } \\
\text { cohort }\end{array}$ & 280 & 98 & 182 & 46 & 52 & $\begin{array}{l}\text { TBI, stroke, } \\
\text { SAH, } \\
\text { ICH }\end{array}$ & $\begin{array}{l}\text { ICH, } \\
\text { infection, } \\
\text { EFC, BFR }\end{array}$ & $\mathrm{ABF}$ & 103 & NS & $6 \star$ \\
\hline Tantawi 2012 & $\begin{array}{l}\text { Retrospective } \\
\text { cohort }\end{array}$ & 200 & 200 & 0 & 25 & 100 & TBI & $\begin{array}{l}\text { Infection, } \\
\text { seizure, } \\
\text { other }\end{array}$ & PMMA, TM & NS & 29 & $6 \star$ \\
\hline Bobinski 2013 & $\begin{array}{l}\text { Retrospective } \\
\text { cohort }\end{array}$ & 49 & 49 & 0 & 43 & 78 & TBI & $\begin{array}{l}\text { ICH, } \\
\text { infection, } \\
\text { BFR, other }\end{array}$ & ABF, PMMA & 114 & 5 & $6 \star$ \\
\hline Dünisch 2013 & $\begin{array}{l}\text { Retrospective } \\
\text { cohort }\end{array}$ & 372 & 134 & 238 & 49 & 57 & $\begin{array}{l}\text { TBI, SAH, } \\
\text { stroke, } \\
\text { ICH, } \\
\text { tumor }\end{array}$ & $\mathrm{BFR}$ & $\mathrm{ABF}$ & $78^{\wedge}$ & 12 & $7 \star$ \\
\hline Lee 2013 & $\begin{array}{l}\text { Retrospective } \\
\text { cohort }\end{array}$ & 236 & 142 & 94 & 38 & 78 & $\begin{array}{l}\text { TBI, ICH, } \\
\text { stroke, } \\
\text { infection, } \\
\text { tumor }\end{array}$ & $\begin{array}{l}\text { Seizures, } \\
\text { infection, } \\
\text { ICH, other }\end{array}$ & $\begin{array}{l}\text { TM, ABF, } \\
\text { PEEK }\end{array}$ & NS & $>12$ & $8 \star$ \\
\hline Oladunjoye 2013 & $\begin{array}{l}\text { Retrospective } \\
\text { cohort }\end{array}$ & 62 & 44 & 18 & NS & 60 & $\begin{array}{l}\text { TBI, stroke, } \\
\text { ICH, } \\
\text { SAH }\end{array}$ & $\begin{array}{l}\mathrm{HC}, \mathrm{EFC}, \\
\quad \text { infections }\end{array}$ & $\mathrm{ABF}$ & $54^{\wedge}$ & NS & $8 \star$ \\
\hline Piedra 2013 & $\begin{array}{l}\text { Retrospective } \\
\text { cohort }\end{array}$ & 74 & 0 & 74 & 47 & 50 & $\begin{array}{l}\text { Stroke, ICH, } \\
\text { VL }\end{array}$ & $\begin{array}{l}\text { ICH, } \\
\text { infection, } \\
\text { HC, BFR }\end{array}$ & $\begin{array}{l}\mathrm{ABF}, \\
\text { synthetic }\end{array}$ & 91 & 13 & $7 \star$ \\
\hline Schuss 2013 & $\begin{array}{l}\text { Retrospective } \\
\text { cohort }\end{array}$ & 254 & 89 & 165 & 45 & 52 & $\begin{array}{l}\text { TBI, stroke, } \\
\text { SAH, } \\
\text { ICH, } \\
\text { other }\end{array}$ & BFR & $\mathrm{ABF}$ & 103 & 12 & $8 \star$ \\
\hline Walcott 2013 & $\begin{array}{l}\text { Retrospective } \\
\text { cohort }\end{array}$ & 239 & 146 & 93 & 42 & 66 & $\begin{array}{l}\text { VL, stroke, } \\
\text { TBI }\end{array}$ & $\begin{array}{l}\text { ICH, } \\
\text { infection, } \\
\text { HC, } \\
\text { seizures }\end{array}$ & $\begin{array}{l}\mathrm{ABF}, \\
\text { synthetic }\end{array}$ & 183 & 15 & $8 \star$ \\
\hline Wachter 2013 & $\begin{array}{l}\text { Retrospective } \\
\text { cohort }\end{array}$ & 136 & 52 & 84 & 45 & 68 & $\begin{array}{l}\text { TBI, stroke, } \\
\text { SAH, } \\
\text { ICH, } \\
\text { infection, } \\
\text { ST, } \\
\text { unknown }\end{array}$ & $\begin{array}{l}\text { BFR, } \\
\text { infection, } \\
\text { ICH, EFC, } \\
\text { other }\end{array}$ & ABF, PMMA & 111 & NS & $7 \star$ \\
\hline Cheng 2014 & $\begin{array}{l}\text { Prospective } \\
\text { cohort }\end{array}$ & 290 & 199 & 91 & 50 & 67 & $\begin{array}{l}\text { TBI, stroke, } \\
\text { ICH, } \\
\text { SAH, } \\
\text { tumor, } \\
\text { other }\end{array}$ & Infection & $\mathrm{ABF}$ & 60 & $6-12$ & $7 \star$ \\
\hline Klinger 2014 & $\begin{array}{l}\text { Retrospective } \\
\text { cohort }\end{array}$ & 249 & 118 & 131 & 44 & 63 & $\begin{array}{l}\text { TBI, SAH, } \\
\text { ICH, } \\
\text { stroke, } \\
\text { infection, } \\
\text { tumor, } \\
\text { other }\end{array}$ & $\begin{array}{l}\text { Infection, } \\
\text { BFR, ICH }\end{array}$ & ABF, acrylic & NS & NS & $7 \star$ \\
\hline Mukherjee 2014 & $\begin{array}{l}\text { Retrospective } \\
\text { cohort }\end{array}$ & 174 & 69 & 105 & 41 & 60 & $\begin{array}{l}\text { TBI, tumor, } \\
\text { infection }\end{array}$ & $\begin{array}{l}\text { Seizures, } \\
\text { ICH, } \\
\text { infection, } \\
\text { other }\end{array}$ & $\mathrm{TM}$ & 311 & NS & $6 \star$ \\
\hline Schoekler 2014 & $\begin{array}{l}\text { Retrospective } \\
\text { cohort }\end{array}$ & 58 & 18 & 40 & 46 & 57 & $\begin{array}{l}\text { Stroke, TBI, } \\
\text { ICH, } \\
\text { tumor }\end{array}$ & BFR & $\begin{array}{l}\text { ABF, PMMA, } \\
\text { PEEK, }\end{array}$ & 233 & 6 & $8 \star$ \\
\hline Sundseth 2014 & & 47 & 0 & 47 & 48 & 57 & & & $\mathrm{ABF}$ & $97^{\wedge}$ & $41^{\wedge}$ & $6 \star$ \\
\hline
\end{tabular}


Table 2 (continued)

\begin{tabular}{|c|c|c|c|c|c|c|c|c|c|c|c|c|}
\hline \multirow[t]{2}{*}{ Reference } & \multirow[t]{2}{*}{ Study design } & \multicolumn{3}{|c|}{$\begin{array}{l}\text { Number of } \\
\text { patients }\end{array}$} & \multirow{2}{*}{$\begin{array}{l}\text { Mean } \\
\text { age } \\
\text { (years) }\end{array}$} & \multirow[t]{2}{*}{$\begin{array}{l}\text { Males } \\
(\%)\end{array}$} & \multirow[t]{2}{*}{$\begin{array}{l}\text { Indications } \\
\text { for DC }\end{array}$} & \multirow[t]{2}{*}{$\begin{array}{l}\text { Described } \\
\text { complications }\end{array}$} & \multirow[t]{2}{*}{$\begin{array}{l}\text { Implant } \\
\text { material }\end{array}$} & \multirow{2}{*}{$\begin{array}{l}\text { Mean } \\
\text { DC to } \\
\text { CP } \\
\text { interval } \\
\text { (days) }\end{array}$} & \multirow{2}{*}{$\begin{array}{l}\text { Follow- } \\
\text { up } \\
\text { (months) }\end{array}$} & \multirow[t]{2}{*}{$\begin{array}{l}\text { Quality } \\
\text { (NOS) }\end{array}$} \\
\hline & & Total & TBI & $\begin{array}{l}\text { Non- } \\
\text { TBI }\end{array}$ & & & & & & & & \\
\hline & $\begin{array}{l}\text { Retrospective } \\
\text { cohort }\end{array}$ & & & & & & $\begin{array}{l}\text { Stroke, ICH, } \\
\text { tumor }\end{array}$ & $\begin{array}{l}\text { Infection, } \\
\text { BFR }\end{array}$ & & & & \\
\hline Brommeland 2015 & $\begin{array}{l}\text { Retrospective } \\
\text { cohort }\end{array}$ & 71 & 58 & 13 & $31^{\wedge}$ & 62 & TBI, stroke & $\begin{array}{l}\text { Infection, } \\
\text { ICH, BFR, } \\
\text { other }\end{array}$ & $\begin{array}{l}\mathrm{ABF}, \\
\text { synthetic }\end{array}$ & $74^{\wedge}$ & $10^{\wedge}$ & $7 \star$ \\
\hline Chen 2015 & $\begin{array}{l}\text { Retrospective } \\
\text { cohort }\end{array}$ & 7 & 7 & 0 & 47 & 71 & TBI & Infection & $\mathrm{TM}$ & 54 & 29 & $7 \star$ \\
\hline Paredes 2015 & $\begin{array}{l}\text { Prospective } \\
\text { cohort }\end{array}$ & 55 & 28 & 27 & 43 & 67 & $\begin{array}{l}\text { TBI, ICH, } \\
\text { VL }\end{array}$ & $\begin{array}{l}\text { Infection, } \\
\text { ICH }\end{array}$ & $\begin{array}{l}\text { ABF, PEEK, } \\
\text { PMMA }\end{array}$ & 309 & NS & $5 \star$ \\
\hline Rosseto 2015 & $\begin{array}{l}\text { Retrospective } \\
\text { cohort }\end{array}$ & 45 & 38 & 7 & 32 & 82 & $\begin{array}{l}\text { Stroke, } \\
\text { infection, } \\
\text { TBI, } \\
\text { unknown }\end{array}$ & Infection & $\begin{array}{l}\mathrm{ABF}, \\
\text { synthetic }\end{array}$ & NS & $2-27$ & $6 \star$ \\
\hline Tsang 2015 & $\begin{array}{l}\text { Retrospective } \\
\text { cohort }\end{array}$ & 162 & 68 & 94 & 49 & 63 & $\begin{array}{l}\text { VL, TBI, } \\
\text { infection, } \\
\text { tumor }\end{array}$ & $\begin{array}{l}\text { Infection, } \\
\text { BFR, } \\
\text { seizures, } \\
\text { other }\end{array}$ & $\begin{array}{l}\text { ABF, acrylic, } \\
\text { TM }\end{array}$ & 162 & 58 & $8 \star$ \\
\hline $\begin{array}{l}\text { Von Der Breile } \\
2015\end{array}$ & $\begin{array}{l}\text { Retrospective } \\
\text { cohort }\end{array}$ & 219 & 159 & 60 & 43 & NS & TBI, stroke & $\begin{array}{l}\text { Infection, } \\
\text { BFR }\end{array}$ & $\begin{array}{r}\text { ABF, PMMA, } \\
\text { customized }\end{array}$ & NS & NS & $7 \star$ \\
\hline Borger 2016 & $\begin{array}{l}\text { Retrospective } \\
\text { cohort }\end{array}$ & 75 & 0 & 75 & 52 & 48 & stroke & $\begin{array}{l}\text { Infection, } \\
\text { ICH, other }\end{array}$ & NS & 145 & NS & $6 \star$ \\
\hline Chaturvedi 2016 & $\begin{array}{l}\text { Retrospective } \\
\text { cohort }\end{array}$ & 74 & 74 & 0 & $32^{\wedge}$ & 71 & TBI & $\begin{array}{l}\text { Infection, } \\
\text { seizure, } \\
\text { ICH, EFC, } \\
\text { other }\end{array}$ & $\begin{array}{c}\mathrm{ABF}, \mathrm{TM}, \\
\text { acrylic }\end{array}$ & 305 & 32 & $6 \star$ \\
\hline Daou 2016 & $\begin{array}{l}\text { Retrospective } \\
\text { cohort }\end{array}$ & 114 & 15 & 99 & 51 & 47 & $\begin{array}{l}\text { SAH, ICH, } \\
\text { stroke, } \\
\text { TBI, ICH, } \\
\text { VL }\end{array}$ & $\begin{array}{l}\text { BFR, HC, } \\
\text { ICH, } \\
\text { seizures, } \\
\text { infection }\end{array}$ & $\mathrm{ABF}$ & 180 & 25 & $8 \star$ \\
\hline Honeybul 2016 & $\begin{array}{l}\text { Retrospective } \\
\text { cohort }\end{array}$ & 512 & 330 & 182 & 39 & 71 & $\begin{array}{l}\text { TBI, stroke. } \\
\text { tumor, } \\
\text { infection, } \\
\text { SAH, } \\
\text { ICH }\end{array}$ & $\begin{array}{l}\text { EFC, ICH, } \\
\text { seizures, } \\
\text { infection, } \\
\text { BFR }\end{array}$ & $\begin{array}{l}\text { ABF, TM, } \\
\text { mesenchy- } \\
\text { mal stromal } \\
\text { cells }\end{array}$ & 98 & NS & $7 \star$ \\
\hline Krause-Titz 2016 & $\begin{array}{l}\text { Retrospective } \\
\text { cohort }\end{array}$ & 248 & 80 & 168 & 46 & 51 & $\begin{array}{l}\text { TBI, SAH, } \\
\text { stroke, } \\
\text { tumor }\end{array}$ & $\begin{array}{l}\text { HC, seizures, } \\
\text { ICH, } \\
\text { infection, } \\
\text { other }\end{array}$ & $\begin{array}{c}\text { ABF, PMMA, } \\
\text { PMMA+ } \\
\text { TM, } \\
\text { customized }\end{array}$ & 231 & NS & $7 \star$ \\
\hline Pierson 2016 & $\begin{array}{l}\text { Retrospective } \\
\text { cohort }\end{array}$ & 39 & 24 & 15 & 57 & 61 & $\begin{array}{l}\text { TBI, VL, } \\
\text { ICH, } \\
\text { stroke, } \\
\text { infection, } \\
\text { tumor }\end{array}$ & $\begin{array}{l}\text { Infection, } \\
\text { seizures }\end{array}$ & $\begin{array}{l}\mathrm{ABF} \\
\quad \text { customized }\end{array}$ & 124 & NS & $7 \star$ \\
\hline Quah 2016 & $\begin{array}{l}\text { Prospective } \\
\text { cohort }\end{array}$ & 70 & 47 & 23 & 40 & 71 & $\begin{array}{l}\text { TBI, ICH, } \\
\text { stroke, } \\
\text { other }\end{array}$ & Infection & $\begin{array}{l}\text { ABF, TM, } \\
\text { acrylic, } \\
\text { PEEK }\end{array}$ & NS & 23 & $5 \star$ \\
\hline Riordan 2016 & $\begin{array}{l}\text { Retrospective } \\
\text { cohort }\end{array}$ & 186 & 92 & 94 & 36 & 62 & $\begin{array}{l}\text { TBI, SAH, } \\
\text { infection, } \\
\text { ICH, } \\
\text { tumor, } \\
\text { stroke }\end{array}$ & Infection & $\begin{array}{l}\mathrm{ABF}, \\
\text { synthetic }\end{array}$ & 220 & NS & $7 \star$ \\
\hline Roberts 2016 & $\begin{array}{l}\text { Retrospective } \\
\text { cohort }\end{array}$ & 14 & 14 & 0 & 24 & 100 & TBI & Infection & $\begin{array}{l}\mathrm{ABF}, \\
\text { synthetic }\end{array}$ & NS & NS & $5 \star$ \\
\hline Shibahashi 2017 & $\begin{array}{l}\text { Retrospective } \\
\text { cohort }\end{array}$ & 155 & 82 & 73 & $57^{\wedge}$ & 59 & $\begin{array}{c}\text { TBI, SAH, } \\
\text { ICH, } \\
\text { stroke }\end{array}$ & $\begin{array}{l}\text { Infection, } \\
\text { ICH }\end{array}$ & $\begin{array}{l}\mathrm{ABF}, \\
\text { synthetic }\end{array}$ & 44 & 6 & $8 \star$ \\
\hline $\begin{array}{l}\text { Abode-Iamah } \\
2018\end{array}$ & $\begin{array}{l}\text { Retrospective } \\
\text { cohort }\end{array}$ & 258 & 139 & 119 & 49 & 62 & & $\begin{array}{l}\text { Infection, } \\
\text { seizures, }\end{array}$ & $\begin{array}{l}\mathrm{ABF}, \\
\text { synthetic }\end{array}$ & NS & NS & $7 \star$ \\
\hline
\end{tabular}


Table 2 (continued)

\begin{tabular}{|c|c|c|c|c|c|c|c|c|c|c|c|c|}
\hline \multirow[t]{2}{*}{ Reference } & \multirow[t]{2}{*}{ Study design } & \multicolumn{3}{|c|}{$\begin{array}{l}\text { Number of } \\
\text { patients }\end{array}$} & \multirow{2}{*}{$\begin{array}{l}\text { Mean } \\
\text { age } \\
\text { (years) }\end{array}$} & \multirow[t]{2}{*}{$\begin{array}{l}\text { Males } \\
(\%)\end{array}$} & \multirow[t]{2}{*}{$\begin{array}{l}\text { Indications } \\
\text { for DC }\end{array}$} & \multirow[t]{2}{*}{$\begin{array}{l}\text { Described } \\
\text { complications }\end{array}$} & \multirow[t]{2}{*}{$\begin{array}{l}\text { Implant } \\
\text { material }\end{array}$} & \multirow{2}{*}{$\begin{array}{l}\text { Mean } \\
\text { DC to } \\
\mathrm{CP} \\
\text { interval } \\
\text { (days) }\end{array}$} & \multirow{2}{*}{$\begin{array}{l}\text { Follow- } \\
\text { up } \\
\text { (months) }\end{array}$} & \multirow[t]{2}{*}{$\begin{array}{l}\text { Quality } \\
\text { (NOS) }\end{array}$} \\
\hline & & Total & TBI & $\begin{array}{l}\text { Non- } \\
\text { TBI }\end{array}$ & & & & & & & & \\
\hline & & & & & & & $\begin{array}{l}\text { ICH, TBI, } \\
\text { tumor, } \\
\text { other }\end{array}$ & $\begin{array}{l}\mathrm{HC}, \mathrm{BFR}, \\
\mathrm{EFC}, \mathrm{ICH}\end{array}$ & & & & \\
\hline Jin 2018 & $\begin{array}{l}\text { Retrospective } \\
\text { cohort }\end{array}$ & 57 & 36 & 21 & 43 & 70 & $\begin{array}{c}\text { TBI, stroke, } \\
\text { SAH, } \\
\text { ICH, } \\
\text { tumor }\end{array}$ & $\begin{array}{l}\text { Infection, } \\
\text { BFR }\end{array}$ & $\mathrm{ABF}$ & 136 & 43 & $7 \star$ \\
\hline Morton 2018 & $\begin{array}{l}\text { Retrospective } \\
\text { cohort }\end{array}$ & 754 & 388 & 366 & 44 & 60 & $\begin{array}{l}\text { TBI, ICH, } \\
\text { SAH, } \\
\text { stroke, } \\
\text { infection, } \\
\text { tumor }\end{array}$ & $\begin{array}{l}\text { HC, BFR, } \\
\text { seizures, } \\
\text { ICH, other }\end{array}$ & $\begin{array}{l}\mathrm{ABF}, \\
\text { synthetic }\end{array}$ & NS & 8 & $8 \star$ \\
\hline Posti 2018 & $\begin{array}{l}\text { Retrospective } \\
\text { cohort }\end{array}$ & 155 & 40 & 115 & 43 & 66 & $\begin{array}{l}\text { TBI, stroke, } \\
\text { tumor, } \\
\text { infection, } \\
\text { ICH, } \\
\text { SAH, } \\
\text { other }\end{array}$ & NS & $\begin{array}{l}\text { ABF, TM, } \\
\text { HA, } \\
\text { FRC-BG, } \\
\text { PMMA, } \\
\text { PEEK, PE }\end{array}$ & 330 & 12 & $8 \star$ \\
\hline Kim 2019 & $\begin{array}{l}\text { Retrospective } \\
\text { cohort }\end{array}$ & 126 & 54 & 72 & 51 & 63 & $\begin{array}{l}\text { SAH, tumor, } \\
\text { stroke, } \\
\text { ICH, TBI }\end{array}$ & $\begin{array}{l}\text { Infection, } \\
\text { BFR }\end{array}$ & $\mathrm{ABF}$ & NS & NS & $6 \star$ \\
\hline Shih 2019 & $\begin{array}{l}\text { Retrospective } \\
\text { cohort }\end{array}$ & 189 & 113 & 76 & $52^{\wedge}$ & 67 & $\begin{array}{l}\text { TBI, stroke, } \\
\text { tumor, } \\
\text { infection }\end{array}$ & $\begin{array}{l}\text { Seizures, } \\
\text { ICH, } \\
\text { infection, } \\
\text { other }\end{array}$ & $\begin{array}{l}\text { ABF, PMMA, } \\
\text { TM }\end{array}$ & 52 & 24 & $8 \star$ \\
\hline Yeap 2019 & $\begin{array}{l}\text { Retrospective } \\
\text { cohort }\end{array}$ & 336 & 220 & 116 & 45 & 67 & $\begin{array}{l}\text { ICH, VL, } \\
\text { stroke, } \\
\text { infection, } \\
\text { TBI, } \\
\text { tumor }\end{array}$ & Seizures & $\begin{array}{c}\mathrm{ABF}, \mathrm{TM}, \\
\text { PMMA }\end{array}$ & 312 & NS & $7 \star$ \\
\hline Alkhaibary 2020 & $\begin{array}{l}\text { Retrospective } \\
\text { cohort }\end{array}$ & 103 & 78 & 25 & 31 & 84 & $\begin{array}{l}\text { TBI, stroke, } \\
\text { SAH, } \\
\text { other }\end{array}$ & Infection & $\mathrm{ABF}$ & 115 & 7 & $8 \star$ \\
\hline Goedemans 2020 & $\begin{array}{l}\text { Retrospective } \\
\text { cohort }\end{array}$ & 145 & 27 & 118 & 44 & 46 & $\begin{array}{l}\text { Stroke, } \\
\text { SAH, } \\
\text { TBI, ST, } \\
\text { ICH, } \\
\text { infection, } \\
\text { tumor }\end{array}$ & $\begin{array}{l}\mathrm{ICH}, \\
\text { infection, } \\
\text { EFC, HC }\end{array}$ & $\begin{array}{l}\mathrm{ABF}, \\
\text { synthetic }\end{array}$ & 136 & 12 & $8 \star$ \\
\hline Rashidi 2020 & $\begin{array}{l}\text { Retrospective } \\
\text { cohort }\end{array}$ & 303 & 110 & 193 & 51 & 59 & $\begin{array}{l}\text { TBI, stroke, } \\
\text { ICH, } \\
\text { SAH, } \\
\text { tumor, } \\
\text { infection, } \\
\text { other }\end{array}$ & BFR & $\mathrm{ABF}$ & 182 & 13 & $8 \star$ \\
\hline Our series & $\begin{array}{l}\text { Retrospective } \\
\text { cohort }\end{array}$ & 149 & 56 & 93 & 53 & 57 & $\begin{array}{l}\text { TBI, } \\
\text { infection, } \\
\text { tumor } \\
\text { stroke, } \\
\text { ICH }\end{array}$ & $\begin{array}{l}\text { Infection, } \\
\text { seizure, } \\
\text { HC, ICH, } \\
\text { BFR }\end{array}$ & $\begin{array}{l}\mathrm{ABF}, \\
\text { synthetic }\end{array}$ & 159 & 77 & $8 \star$ \\
\hline
\end{tabular}

$T B I$ traumatic brain injury, $D C$ decompressive craniectomy, $C P$ cranioplasty, NOS Newcastle-Ottawa Scale, $S A H$ subarachnoid hemorrhage, $S T$ sinus thrombosis, $V L$ venous lesion, $I C H$ intracranial hemorrhage, $B F R$ bone flap resorption, $E F C$ extra-axial fluid collection, $H C$ hydrocephalus, $N S$ not specified, $A B F$ autologous bone flap, $P M M A$ polymethylmethacrylate, $T M$ titanium mesh, $H A$ hydroxyapatite, $F R C$ - $B G$ fiber-reinforced compositebioactive glass, $P E E K$ polyetheretherketone, $P E$ polyethylene, $N / A$ not applicable

*Data extracted from Cheng et al. [31] (original article in Japanese); ${ }^{\wedge}$ median 
The rates of individual complications in our institution were $0.7 \%$ for new-onset seizures, $7.4 \%$ for $\mathrm{ICH}, 22.1 \%$ for infection, $8.7 \%$ for hydrocephalus, and $17.1 \%$ for BFR. When subdivided according to complication type, no single complication had significantly different odds in TBI patients compared with non-TBI ones.

\section{Overall complications}

In total, 23 studies reported the overall rates of post-CP complications seen in their cohorts. These included seizures $(n=5)$, ICH ( $n=13)$, EFC ( $n=7)$, infection ( $n=19$ studies), hydrocephalus $(n=5)$, BFR $(n=8)$, and other complications $(n=6)$. Three studies did not specify which complications were considered.

The pooled overall complication rate of CP was $24.6 \%$ (683/2779) when considering all indications together, consisting of $27.8 \%(353 / 1269)$ in TBI patients and $21.9 \%$ $(330 / 1510)$ in non-TBI patients.

Sixteen of the studies reporting overall complication rates allowed for comparison between the TBI and non-TBI patients (Fig. 2) (while the other 7 only reported complication rates for a single primary indication). The pooled rate of overall complications from these studies was $24.0 \%(n=549$ / 2283), ranging from 14.6 to $42.3 \%$.

No significant difference in the odds of overall complications was found in TBI patients $(n=254 / 969$ procedures, $26.2 \%)$ when compared with non-TBI patients $(n=295 /$ $1314,22.5 \%$; OR 1.26, CI $0.88-1.82$, and $p=0.20$ ) using a random-effects model $\left(I^{2}=62 \%\right.$ and $\left.p=0.0005\right)$. Similar results were obtained when the analysis was conducted without adding our series to the published data (Supplementary Fig. S2).

\section{Seizures}

In total, 10 studies reported new-onset seizure rates. The pooled rate was $13.2 \%$ (307/2333). When divided according to initial indication, the rate of seizure was $12.6 \%(177 / 1405)$ in TBI patients and 14.0\% (130/928) in non-TBI patients.

Seven of the studies reporting seizure rates allowed for comparison between TBI and non-TBI patients (Fig. 3). The pooled rate of seizures from these studies was $14.6 \%(n=284 /$ 1951), ranging from 0.7 to $29.0 \%$.

There was no difference in the odds of seizure in TBI patients $(n=154 / 1023$ procedures, $15.1 \%)$ when compared with non-TBI patients $(n=130 / 928,14.0 \%$; OR 0.96 , CI 0.64 1.44 , and $p=0.86)$ using a random-effects model $\left(I^{2}=43 \%\right.$ and $p=0.10$ ). This was true with (Fig. 3) or without the addition of the cases of our series (Supplementary Fig. S3).

\section{Intracranial hemorrhage}

In total, 10 studies reported ICH rates. The pooled rate was $4.8 \%(63 / 1320), 4.6 \%(28 / 615)$ in TBI patients, and 5.0\% (35/ $705)$ in non-TBI patients.

Five of the studies reporting ICH allowed for comparison between TBI and non-TBI patients (Fig. 4). One study reported postoperative epidural hematomas only, while the others, including ours, considered both epidural and subdural hematomas requiring evacuation. One study also considered subgaleal hematomas.

The pooled rate of hemorrhage from these studies was $5.0 \%(n=46 / 913)$, ranging from 3.6 to $7.4 \%$.

There was no difference in the odds of hemorrhage in TBI patients ( $n=17 / 357$ procedures, $4.8 \%)$ when compared with non-TBI patients $(n=29 / 556,5.2 \%$; OR 0.98 , CI $0.52-1.83$, and $p=0.95)$ using a random-effects model $\left(I^{2}=0 \%\right.$ and $p=$

\begin{tabular}{|c|c|c|c|c|c|c|c|}
\hline \multirow[b]{2}{*}{ Study or Subgroup } & \multicolumn{2}{|l|}{ TBI } & \multicolumn{2}{|c|}{ Non-TBI } & \multicolumn{3}{|c|}{ Odds Ratio } \\
\hline & Events & Total & Events & Total & Weight & $\mathrm{M}-\mathrm{H}$, Random, $95 \% \mathrm{Cl}$ & I Year \\
\hline Moreira-Gonzalez 2003 & 25 & 99 & 32 & 101 & $8.2 \%$ & $0.73[0.39,1.35]$ & 2003 \\
\hline Kriegel 2007 & 2 & 11 & 10 & 28 & $3.1 \%$ & $0.40[0.07,2.23]$ & 2007 \\
\hline Chang 2010 & 7 & 79 & 27 & 133 & $6.6 \%$ & $0.38[0.16,0.92]$ & 2010 \\
\hline Sobani 2011 & 22 & 55 & 12 & 41 & $6.7 \%$ & $1.61[0.68,3.82]$ & 2011 \\
\hline Güresir 2011 & 14 & 74 & 17 & 122 & $7.2 \%$ & $1.44[0.66,3.13]$ & 2011 \\
\hline Schuss 2012 & 20 & 98 & 26 & 182 & $8.0 \%$ & $1.54[0.81,2.93]$ & 2012 \\
\hline Watcher 2013 & 19 & 52 & 22 & 84 & $7.4 \%$ & $1.62[0.77,3.42]$ & 2013 \\
\hline Oladunjoye 2013 & 16 & 44 & 7 & 18 & $5.3 \%$ & $0.90[0.29,2.78]$ & 2013 \\
\hline Mukherjee 2014 & 24 & 69 & 22 & 105 & $7.8 \%$ & $2.01[1.02,3.98]$ & 2014 \\
\hline Von Der Breile 2015 & 30 & 159 & 2 & 60 & $3.9 \%$ & $6.74[1.56,29.17]$ & 2015 \\
\hline Paredes 2015 & 4 & 28 & 6 & 27 & $4.1 \%$ & $0.58[0.14,2.35]$ & 2015 \\
\hline Pierson 2016 & 7 & 24 & 3 & 15 & $3.6 \%$ & $1.65[0.35,7.69]$ & 2016 \\
\hline Posti 2018 & 7 & 40 & 28 & 115 & $6.4 \%$ & $0.66[0.26,1.65]$ & 2018 \\
\hline Kim 2019 & 21 & 54 & 21 & 72 & $7.4 \%$ & $1.55[0.73,3.26]$ & 2019 \\
\hline Goedemans 2020 & 15 & 27 & 18 & 118 & $6.4 \%$ & $6.94[2.80,17.25]$ & 2020 \\
\hline Current Study & 21 & 56 & 42 & 93 & $7.8 \%$ & $0.73[0.37,1.44]$ & 2020 \\
\hline Total $(95 \% \mathrm{Cl})$ & & 969 & & 1314 & $100.0 \%$ & $1.26[0.88,1.82]$ & \\
\hline Total events & 254 & & 295 & & & & \\
\hline \multicolumn{8}{|c|}{ Heterogeneity: $\mathrm{Tau}^{2}=0.32 ; \mathrm{Chi}^{2}=39.69, \mathrm{df}=15(\mathrm{P}=0.0005) ; \mathrm{I}^{2}=62 \%$} \\
\hline Test for overall effect: $Z$ & $27(P=$ & $0.20)$ & & & & & \\
\hline
\end{tabular}

Fig. 2 Forest plot of studies reporting overall cranioplasty complications in TBI and non-TBI patients 


\begin{tabular}{|c|c|c|c|c|c|c|c|c|}
\hline \multirow[b]{2}{*}{ Study or Subgroup } & \multicolumn{2}{|l|}{ TBI } & \multicolumn{2}{|c|}{ Non-TBI } & \multicolumn{3}{|c|}{ Odds Ratio } & \multirow{2}{*}{$\begin{array}{c}\text { Odds Ratio } \\
\text { M-H, Random, } 95 \% \text { Cl } \\
\end{array}$} \\
\hline & Events & Total & Events & Total & Weight & M-H, Random, $95 \% \mathrm{Cl}$ & Year & \\
\hline Lee 2013 & 17 & 142 & 19 & 94 & $17.1 \%$ & $0.54[0.26,1.10]$ & 2013 & \\
\hline Krause-Titz 2016 & 29 & 80 & 43 & 168 & $21.1 \%$ & $1.65[0.93,2.93]$ & 2016 & \\
\hline Pierson 2016 & 1 & 24 & 2 & 15 & $2.4 \%$ & $0.28[0.02,3.43]$ & 2016 & \\
\hline Morton 2018 & 12 & 388 & 19 & 366 & $16.5 \%$ & $0.58[0.28,1.22]$ & 2018 & \\
\hline Yeap 2019 & 63 & 220 & 26 & 116 & $22.7 \%$ & $1.39[0.82,2.35]$ & 2019 & \\
\hline Shih 2019 & 32 & 113 & 20 & 76 & $18.7 \%$ & $1.11[0.57,2.13]$ & 2019 & \\
\hline Current Study & 0 & 56 & 1 & 93 & $1.5 \%$ & $0.55[0.02,13.63]$ & 2020 & \\
\hline Total $(95 \% \mathrm{Cl})$ & & 1023 & & 928 & $100.0 \%$ & $0.96[0.64,1.44]$ & & \\
\hline Total events & 154 & & 130 & & & & & \\
\hline \multicolumn{8}{|c|}{ Heterogeneity: $\mathrm{Tau}^{2}=0.11 ; \mathrm{Chi}^{2}=10.58, \mathrm{df}=6(P=0.10) ; \mathrm{I}^{2}=43 \%$} & $\begin{array}{lccc}0.05 & 0.2 & 1 & 5 \\
\text { Higher Odds Non-TBI } & \text { Higher Odds TBI }\end{array}$ \\
\hline
\end{tabular}

Fig. 3 Forest plot of studies reporting new-onset post-cranioplasty seizures in TBI and non-TBI patients

0.86). This was true with (Fig. 4) or without the addition of the cases of our series (Supplementary Fig. S4).

\section{Extra-axial fluid collections}

In total, 8 studies reported the rates of noninfectious and nonhemorrhagic EFCs. The pooled rate was 5.5\% (57/ $1028), 7.3 \%(42 / 578)$ in TBI patients, and $3.3 \%(15 / 450)$ in non-TBI patients.

Five of the studies reporting EFC were allowed for comparison between the TBI and non-TBI group (Fig. 5). These included epidural and subdural fluid collections ( $n=3$ studies), hygroma $(n=3)$, and CSF fistula $(n=2)$. In our cohort, no patient suffered from EFC after CP.

The pooled rate of EFC from these studies was $6.0 \%(n=$ $38 / 636$ ), ranging from 1.1 to $37.3 \%$.

There was no difference in the odds of EFC in TBI patients ( $n=23 / 279$ procedures, $8.2 \%$ ) when compared with non-TBI patients $(n=15 / 357,4.2 \%$; OR 0.88 , CI 0.40-1.92, and $p=$ $0.75)$ using a random-effects model $\left(I^{2}=2 \%\right.$ and $\left.p=0.39\right)$. This was true with (Fig. 5) or without the addition of the cases of our series (Supplementary Fig. S5).

\section{Infection}

In total, 40 studies reported infection rates. The pooled rate was $10.0 \%(546 / 5461)$, consisting of $11.0 \%(325 / 2960)$ in TBI patients and $8.8 \%(221 / 2501)$ in non-TBI patients. In our series, we considered as complicated by infection every cranioplasty associated with persistent fever, raised inflammatory parameters, and/or local swelling or pus. We also considered infected the skull flaps that resulted positive for microbial growth at the time of revision surgery. If both BFR and infection were present in the same patient, the patient was considered as affected by two complications.

Twenty-nine of the studies reporting infection rates allowed for comparison between TBI and non-TBI patients (Fig. 6). The pooled rate of infection from these studies was $10.0 \%$ ( $n=461 / 4609)$, ranging from 2.0 to $24.4 \%$.

There was a significant increase in the odds of infection in TBI patients ( $n=262 / 2304$ procedures, $11.4 \%$ ) when compared with non-TBI patients $(n=199 / 2305,8.6 \%$; OR 1.38 , CI 1.05-1.80, and $p=0.02)$ using a random-effects model $\left(I^{2}\right.$ $=32 \%$ and $p=0.05$ ). This was true with (Fig. 6) or without the addition of the cases of our series (Supplementary Fig. S6).

\section{Hydrocephalus post-CP}

In total, 5 studies reported rates of hydrocephalus developing after CP. The pooled rate was $12.0 \%$ (155/1287), 8.1\% (46/ $568)$ in TBI patients, and $15.2 \%(109 / 719)$ in non-TBI patients.

Four of the studies reporting post-CP hydrocephalus allowed for comparison between TBI and non-TBI patients (Fig. 7). Three studies, including ours, defined hydrocephalus as requiring the placement of a ventriculoperitoneal shunt, while one considered both cases that required VPS and those that resolved spontaneously.

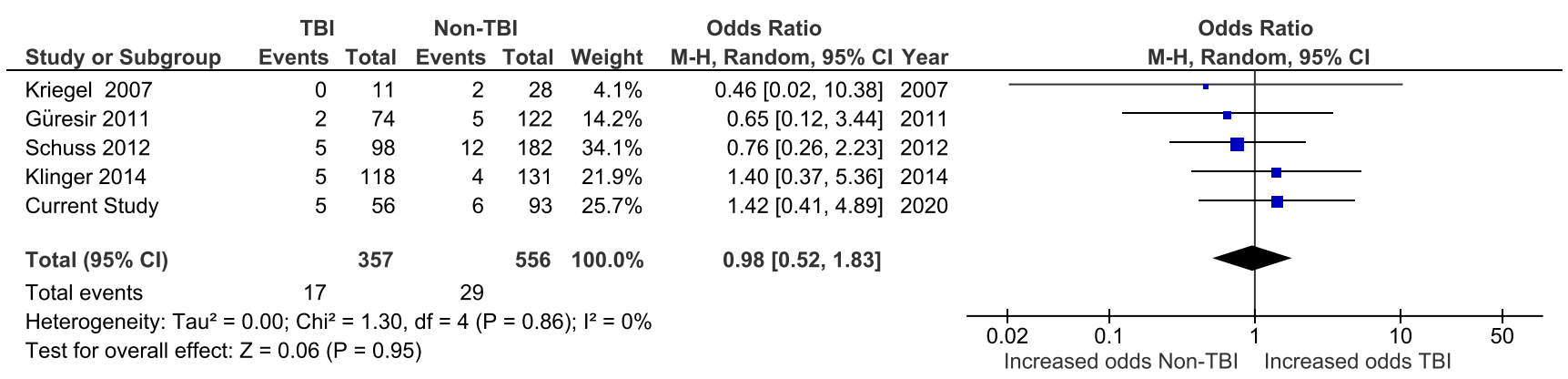

Fig. 4 Forest plot of studies reporting post-cranioplasty intracranial hemorrhage in TBI and non-TBI patients 


\begin{tabular}{|c|c|c|c|c|c|c|c|}
\hline \multirow[b]{2}{*}{ Study or Subgroup } & \multicolumn{2}{|l|}{ TBI } & \multicolumn{2}{|c|}{ Non-TBI } & \multicolumn{2}{|r|}{ Odds Ratio } & \multirow{2}{*}{$\begin{array}{l}\text { Odds Ratio } \\
\text { M-H, Random, } 95 \% \mathrm{Cl} \\
\end{array}$} \\
\hline & Events & Total & Events & Total & Weight & M-H, Random, 95\% Cl Year & \\
\hline Lee 2011 & 14 & 39 & 8 & 20 & $46.5 \%$ & $0.84[0.28,2.54] 2011$ & + \\
\hline Güresir 2011 & 2 & 74 & 1 & 122 & $10.2 \%$ & $3.36[0.30,37.73] 2011$ & \\
\hline Schuss 2012 & 2 & 98 & 1 & 182 & $10.3 \%$ & $3.77[0.34,42.12] 2012$ & \\
\hline Oladunjoye 2013 & 4 & 44 & 4 & 18 & $25.6 \%$ & $0.35[0.08,1.59] 2013$ & \\
\hline Pierson 2016 & 1 & 24 & 1 & 15 & $7.4 \%$ & $0.61[0.04,10.53] 2016$ & \\
\hline Current Study & 0 & 56 & 0 & 93 & & Not estimable 2020 & \\
\hline Total $(95 \% \mathrm{Cl})$ & & 279 & & 357 & $100.0 \%$ & $0.88[0.40,1.92]$ & \\
\hline Total events & 23 & & 15 & & & & \\
\hline $\begin{array}{l}\text { Heterogeneity: } \mathrm{Tau}^{2}= \\
\text { Test for overall effect: }\end{array}$ & $\begin{array}{l}0.02 ; \mathrm{Chi}^{2} \\
Z=0.32(\mathrm{~F}\end{array}$ & $\begin{array}{l}=4.08 \\
P=0.7\end{array}$ & 5) $d f=4(F$ & $=0.39$ & ;) $\left.\right|^{2}=2 \%$ & & $\begin{array}{lllll}1 & 1 & 1 & \\
0.05 & 0.2 & 1 & 5 & 20 \\
\text { Increased odds non-TB| } & \text { Increased odds } & \text { TBI }\end{array}$ \\
\hline
\end{tabular}

Fig. 5 Forest plot of studies reporting post-cranioplasty extra-axial fluid collections in TBI and non-TBI patients

The pooled rate of hydrocephalus from these studies was $12.7 \%(n=154 / 1213)$, ranging from 8.7 to $25.0 \%$.

There was no difference in the odds of hydrocephalus in TBI patients ( $n=46 / 568$ procedures, $8.1 \%$ ) when compared with non-TBI patients $(n=108 / 645,16.7 \%$; OR 0.57 , CI $0.27-1.19$, and $p=0.13)$ using a random-effects model $\left(I^{2}=\right.$ $63 \%$ and $p=0.04)$, when cases collected from the literature and our own were considered together (Fig. 7). However, when published cases were analyzed separately, the odds of hydrocephalus in TBI patients were 7.8\% ( $n=40 / 152)$, compared with $18.3 \%(n=101 / 552)$ in non-TBI patients, giving an odds ratio of 0.43 (CI $0.22-0.86$ ). These results were significant $(p=0.02)$ (Supplementary Fig. S7).

\section{Bone flap resorption}

In total, 13 studies reported BFR rates. For calculation purposes, studies that included synthetic implants in their cohorts were considered only if it was possible to extract the number of patients who underwent $\mathrm{CP}$ using autologous bone graft. Resorption was determined either by clinical exam (softness of the reimplanted flap at palpation and or flap loosening) or imaging (CT scan) or both. In our own series, bone flap resorption was defined as any bone defect superior to $0.5 \mathrm{~cm}$ in its largest diameter when a late CT scan (obtained at least 2 months after CP) was compared to an early (within $24 \mathrm{~h}$ ) postCP CT scan. The pooled rate was $14.2 \%$ (271/1913). When

\begin{tabular}{|c|c|c|c|c|c|c|c|c|c|c|c|c|}
\hline \multirow[b]{2}{*}{ Study or Subgroup } & \multicolumn{2}{|l|}{ TBI } & \multicolumn{2}{|c|}{ Non-TBI } & \multicolumn{3}{|c|}{ Odds Ratio } & \multirow{2}{*}{\multicolumn{5}{|c|}{$\begin{array}{c}\text { Odds Ratio } \\
\text { M-H, Random, } 95 \% \mathrm{Cl} \\
\end{array}$}} \\
\hline & Events & Total & Events & Total & Weight & M-H, Random, 95\% Cl & Year & & & & & \\
\hline Shimizu 2002 & 5 & 29 & 1 & 41 & $1.3 \%$ & $8.33[0.92,75.65]$ & 2002 & & & & & \\
\hline Nagayama 2002 & 4 & 28 & 4 & 167 & $2.7 \%$ & $6.79[1.59,28.97]$ & 2002 & & & & & \\
\hline Iwama 2003 & 1 & 15 & 0 & 34 & $0.7 \%$ & $7.14[0.27,185.73]$ & 2003 & & & & & \\
\hline Matsuno 2006 & 12 & 64 & 13 & 142 & $5.6 \%$ & $2.29[0.98,5.35]$ & 2006 & & & & & \\
\hline Kriegel 2007 & 1 & 11 & 3 & 28 & $1.2 \%$ & $0.83[0.08,9.00]$ & 2007 & & & & & \\
\hline Cheng 2008 & 7 & 60 & 2 & 24 & $2.2 \%$ & $1.45[0.28,7.55]$ & 2008 & & & & & \\
\hline Inamasu 2010 & 4 & 33 & 3 & 37 & $2.4 \%$ & $1.56[0.32,7.57]$ & 2010 & & & & & \\
\hline Güresir 2011 & 11 & 74 & 6 & 122 & $4.4 \%$ & $3.38[1.19,9.56]$ & 2011 & & & & & \\
\hline Archavalis et al. 2012 & 5 & 51 & 16 & 149 & $4.3 \%$ & $0.90[0.31,2.60]$ & 2012 & & & & & \\
\hline Im 2012 & 8 & 61 & 6 & 70 & $4.0 \%$ & $1.61[0.53,4.93]$ & 2012 & & & & & \\
\hline Schuss 2012 & 12 & 98 & 8 & 182 & $5.0 \%$ & $3.03[1.20,7.70]$ & 2012 & & & & & \\
\hline Walcott 2013 & 13 & 146 & 16 & 93 & $6.0 \%$ & $0.47[0.21,1.03]$ & 2013 & & & & & \\
\hline Oladunjoye 2013 & 4 & 44 & 0 & 18 & $0.8 \%$ & $4.11[0.21,80.38]$ & 2013 & & & & & \\
\hline Mukherjee 2014 & 9 & 69 & 7 & 105 & $4.4 \%$ & $2.10[0.74,5.93]$ & 2014 & & & & & \\
\hline Klinger 2014 & 10 & 118 & 5 & 131 & $4.0 \%$ & $2.33[0.77,7.04]$ & 2014 & & & & & \\
\hline Cheng 2014 & 34 & 199 & 12 & 91 & $6.6 \%$ & $1.36[0.67,2.76]$ & 2014 & & & & & \\
\hline Tsang 2015 & 2 & 68 & 11 & 94 & $2.5 \%$ & $0.23[0.05,1.07]$ & 2015 & & & & & \\
\hline Rosseto 2015 & 9 & 38 & 2 & 7 & $1.9 \%$ & $0.78[0.13,4.70]$ & 2015 & & & & & \\
\hline Von Der Breile 2015 & 9 & 159 & 1 & 60 & $1.5 \%$ & $3.54[0.44,28.56]$ & 2015 & & & & & \\
\hline Pierson 2016 & 2 & 24 & 0 & 15 & $0.7 \%$ & $3.44[0.15,76.81]$ & 2016 & & & & & \\
\hline Honeybul 2016 & 28 & 330 & 14 & 182 & $7.0 \%$ & $1.11[0.57,2.17]$ & 2016 & & & & & \\
\hline Riordan 2016 & 22 & 93 & 21 & 87 & $6.8 \%$ & $0.97[0.49,1.93]$ & 2016 & & & & & \\
\hline Quah 2016 & 2 & 47 & 1 & 23 & $1.1 \%$ & $0.98[0.08,11.38]$ & 2016 & & & & & \\
\hline Shibahashi 2017 & 8 & 82 & 5 & 73 & $3.8 \%$ & $1.47[0.46,4.71]$ & 2017 & & & & & \\
\hline $\operatorname{Jin} 2018$ & 3 & 36 & 4 & 21 & $2.3 \%$ & $0.39[0.08,1.93]$ & 2018 & & & & & \\
\hline Abode-lamah 2018 & 9 & 139 & 6 & 119 & $4.2 \%$ & $1.30[0.45,3.78]$ & 2018 & & & & & \\
\hline Kim 2019 & 7 & 54 & 4 & 72 & $3.3 \%$ & $2.53[0.70,9.14]$ & 2019 & & & & & \\
\hline Current Study & 10 & 56 & 23 & 93 & $5.7 \%$ & $0.66[0.29,1.52]$ & 2020 & & & & & \\
\hline Alkhaibary 2020 & 11 & 78 & 5 & 25 & $3.7 \%$ & $0.66[0.20,2.11]$ & 2020 & & & & & \\
\hline Total $(95 \% \mathrm{Cl})$ & & 2304 & & 2305 & $100.0 \%$ & $1.38[1.05,1.80]$ & & & & & & \\
\hline Total events & 262 & & 199 & & & & & & & & & \\
\hline Heterogeneity: $\mathrm{Tau}^{2}=$ & ; $\mathrm{Chi}^{2}=$ & $=41.15$, & $\mathrm{df}=28$ & $(P=0.0$ & $05) ;\left.\right|^{2}=3$ & & & 0.0 & 0.1 & 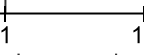 & & 100 \\
\hline
\end{tabular}

Fig. 6 Forest plot of studies reporting post-cranioplasty infection in TBI and non-TBI patients 


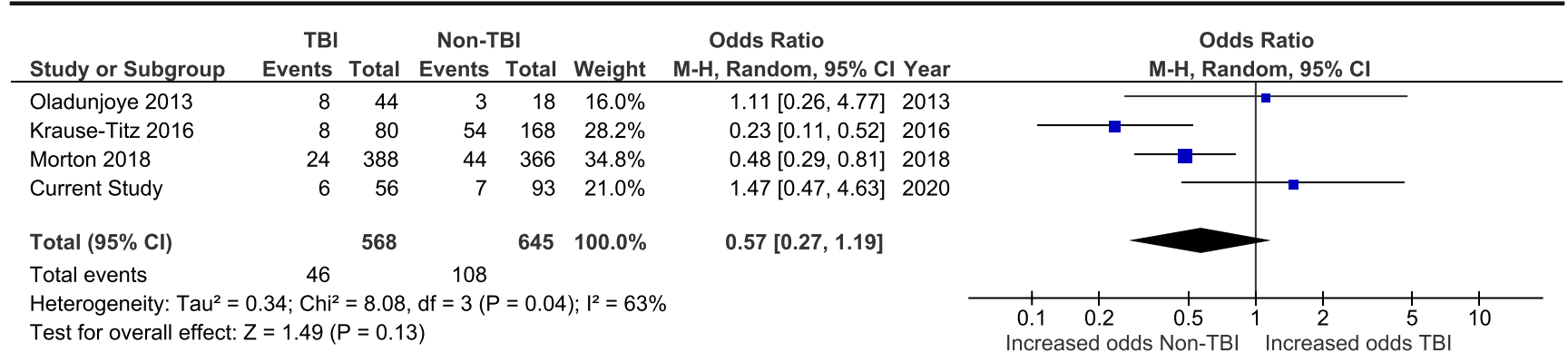

Fig. 7 Forest plot of studies reporting post-cranioplasty hydrocephalus in TBI and non-TBI patients

divided according to initial indication, the rate of BFR was $19.7 \%(165 / 837)$ in TBI patients and 9.8\% (106/1082) in nonTBI patients.

Ten of the studies reporting BFR rates allowed for comparison between TBI and non-TBI patients (Fig. 8). The pooled rate of BFR was $14.8 \%$ ( $n=261 / 1768)$, ranging from 3.9 to $33.3 \%$. There was a significant increase in the odds of resorption in TBI patients $(n=159 / 807$ procedures, $19.7 \%)$ when compared with non-TBI patients $(n=102 / 961,10.6 \%$; OR 1.76 , CI $1.30-2.39$, and $p=0.0003$ ) using a random-effects model $\left(I^{2}=0 \%\right.$ and $\left.p=0.53\right)$. This was true with (Fig. 8) or without the addition of the cases of our series (Supplementary Fig. S8).

\section{Other complications}

Ten studies reported various MCs, which included contour abnormalities/poor cosmesis ( $n=3$ studies) [51, 69, 71], implant displacement $(n=6)[24,25,27,47,70,71]$, implant loosening $(n=2)[44,64]$, and implant fracture $(n=1)$ [44]. The pooled rate of MCs was $3.1 \%$, ranging from 0.4 to $10.0 \%$. Cerebral edema was reported in 2 studies [22, 51], with a pooled rate of $1.07 \%$, ranging from 1.0 to $1.1 \%$. Five studies reported NDs, other than seizures, following CP, including reduced consciousness levels $(n=1)$ [70], urinary retention $(n=1)$ [51], cerebral contusion $(n=1)$ [71], new transient NDs $(n=1)$ [66], and worsening of previous NDs $(n=1)$ [29]. The pooled rate of NDs was $1.9 \%$, ranging from 0.6 to
$6.8 \%$. The reported data for these complications were insufficient for comparison between TBI and non-TBI patients.

\section{Discussion}

This study found no difference in overall complication rates between TBI and non-TBI patients. The heterogeneity of the data was high, indicating that there is a large disagreement between the different studies. This can be explained by several considerations.

First, as can be seen from the table summarizing the study characteristics of the different cohorts (Table 2), the definition of "complications" is quite broad. Some studies measured only complications requiring new surgery [26, 54], while others, as we did in our series, also considered those that could be treated conservatively [28, 35, 42, 51, 56, 57, 62, 79]. Some studies included such outcomes as poor cosmesis [51, 71] or death [56] in their count of complications, while others did not.

Second, comorbidities, choice of graft material, timing of $\mathrm{CP}$, etc. all influence the measured complication rates in a cohort $[42,47,50,72]$. Since the comparison was made between studies done in different locations, at different times, and on different cohorts, these factors inevitably affected the calculated complication rates.

Third, as can be seen from the subgroup analysis of individual complications, TBI might be a risk factor for some

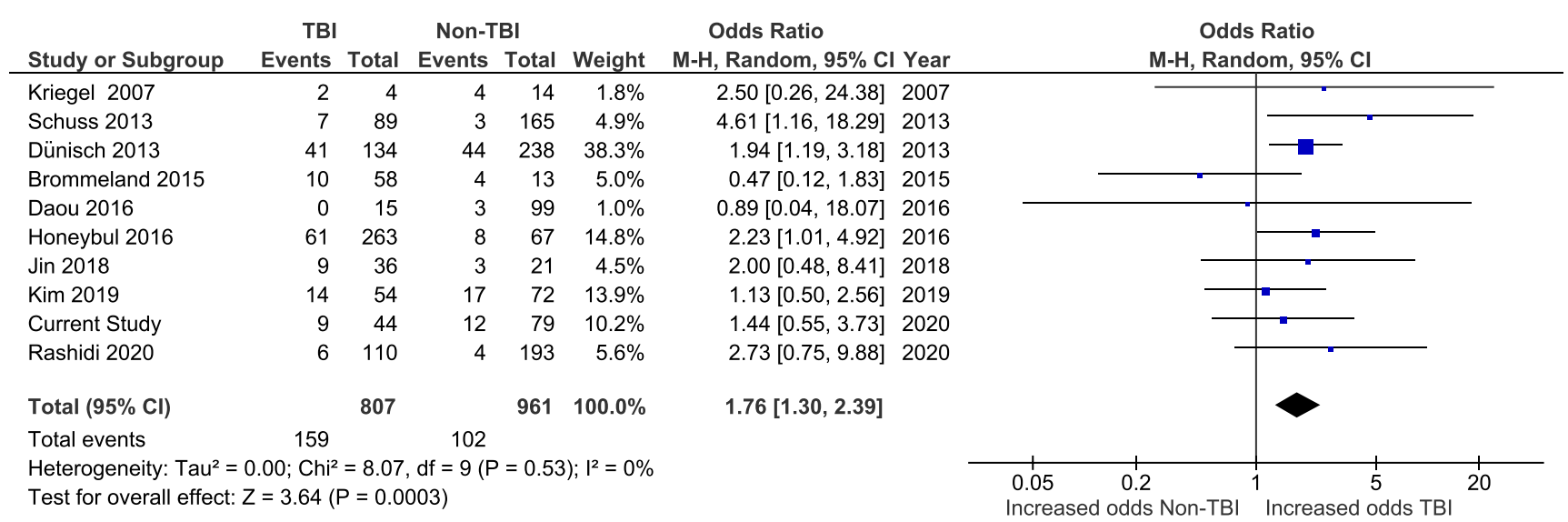

Fig. 8 Forest plot of studies reporting post-cranioplasty bone flap resorption in TBI and non-TBI patients 
complications, such as infections and bone flap resorption, and not for others, such as hydrocephalus developing post$\mathrm{CP}$. Thus, when grouping the complications together, the different effects tended to cancel each other.

On the other hand, when individual complications were analyzed, we found that TBI increased the odds of the following specific complications: infection and autologous bone flap resorption. This was true both with and without our series included in the data (Supplementary Fig. S6 and S8). Conversely, the risk of post-CP hydrocephalus resulted significantly higher in non-TBI patients when only published data were analyzed (Supplementary Fig. S7), but not when we added our data (Fig. 7). A possible explanation is that, compared to other complications, only three studies [44, 50, 53] report data on the incidence of hydrocephalus after $\mathrm{CP}$. This relative scarcity of data enhances sensitivity of the statistical analysis to the addition of our series, suggesting that more data will be necessary to clarify the issue.

Our finding of increased odds of autologous bone flap resorption in TBI patients compared to non-TBI patients is in agreement with previous observations based on a smaller number of patients $[16,80]$. One explanation is that severe trauma is associated with multiple fractures within the reimplanted autologous bone [16]. The quantity and complexity of the fractures may interfere with the osteointegration of the graft and eventually lead to its resorption [81, 82]. Moreover, bone defects resulting from DC after TBI tend to be larger than those resulting from DC performed for other reasons and may therefore hinder the reintegration of the autologous bone flap after CP [57]. Of note, 12 out of the 13 studies examined in the meta-analysis for BFR (representing $96 \%$ of the comparison population) utilized exclusively, as we did, the cryopreservation method to preserve the flaps before $\mathrm{CP}$. Therefore, while it is possible that the method of autologous flap preservation may influence the rates of BFR, this factor was controlled for in our meta-analysis.

Interestingly, our finding in adults is also confirmed in the pediatric population, where patients who undergo $\mathrm{CP}$ with autologous bone flap replacement after DC for TBI have been shown to experience even higher rates of BFR, reaching up to $50.0 \%$ of cases [83].

The association between DC for TBI and higher post-CP infection rates is also supported by several published studies $[23,51,67,84]$. Plausible risk factors predisposing TBI patients for infection may include initial contamination of the skull through traumatic discontinuities of the overlying skin and galea or the presence of multiple scars of traumatic and surgical origin which compromise the vascularization of the surgical flap, resulting in delayed wound healing after CP [62, 85]. Here as well, the larger skull defects following DC after TBI and their association with delayed integration of the reimplanted bone flap may also play a role [57]. Lastly, the possible involvement of the air cavities of the skull, involved by fractures or by the decompressive flap, may also increase the infection rate of the reimplanted bone after CP [43].

Our meta-analysis, including our own patient cohort, did not find significant differences in the odds of hydrocephalus, $\mathrm{ICH}$, insurgence of EFCs, or seizures after CP in TBI patients compared with patients undergoing the same surgery for nonTBI pathologies. Hydrocephalus after $\mathrm{CP}$ has been associated with nontraumatic SAH $[44,50]$, and the incidence of ICH after $\mathrm{CP}$ is similar in patients with different initial neurologic insults [50]. The pooled rate of EFC in the present analysis was $5.5 \%$ (7.3\% in TBI versus $3.3 \%$ in non-TBI). Kurland et al. reported similar results, with a rate of $5.8 \%$ for subdural effusions/hygroma and $6.8 \%$ for CSF leaks/fistulas, for an overall rate of $6.1 \%[86]$.

One retrospective study showed a significant reduction in odds of overall complication rates in TBI patients [28]. The authors attributed their results to the fact that TBI patients in their cohort were significantly younger on average than patients with other primary pathologies. Although patients affected by TBI were younger than non-TBI patients in our study as well, we did not find a significant negative correlation between TBI and odds of post-CP hydrocephalus.

Out of the studies reporting infection, 19 performed regression analysis to compare between autologous bone and synthetic materials for difference in infection rates. Out of these, only one [48] found significantly lower rates of infection in titanium mesh implants when compared to autologous bone. All the rest found no statistical difference in infection rates. This was confirmed recently by a large meta-analysis [87]. However, most of studies used for the meta-analysis did not specify which type of material was used for each patient. It was therefore impossible for us to determine the ratio of autologous cranioplasties between the TBI and non-TBI patients. In our own series, there was no significant difference in the ratio of cranioplasty using autologous bone flap between the TBI group and the non-TBI group $\left(\chi^{2}, 0.979\right.$; DF, $1 ; p<0.3223)$.

Finally, we did not find any significant difference in the timing of cranioplasty between TBI and non-TBI patients. Thus, the DC to CP interval does not appear to have influenced the results of our analysis. This conclusion is, in fact, supported both by our own experience and by that of others [62].

\section{Strengths and limitations}

To the best of our knowledge, this is the only meta-analysis to date exploring the differences in both overall and seven specific post-CP complications between TBI patients and other primary pathologies. Kurland et al. had previously considered the rates of post-CP complications according to different DC indications, including trauma, but their study was only a 
systematic review, utilizing data pooled from different reports to produce a single large cohort [86]. The current study compared between the different cohorts, giving more weight to larger, less biased ones, and attempting to account for the heterogeneity of data using statistical models. In addition, our study is more specific in its categorization of complications into subgroups and analyzed some complications, like seizures, that were not accounted for by Kurland et al.

However, there are several limitations to our analysis. First, the definition of "craniectomy" varied across studies. While studies in which a significant portion of patients underwent craniectomies for purposes other than the reduction of intracranial pressure (e.g., removal of a skull tumor) were excluded, in few cases, it was impossible to quantify the percentage of non-decompressive craniectomies as the reason for craniectomy was not explicitly stated in the paper [32, 48].

Second, as previously stated, the complications controlled for by the individual studies varied significantly. In addition, the definitions for some of the complications differed between the authors, particularly for infection. Some studies only considered infection a complication if it required reoperation [52], whereas others, such as ours, also included infections treated conservatively. Some studies distinguished between surgical site infections, graft infections, and abscesses [66], while others grouped all under the general definition of infection [56]. Cases of cellulitis, meningitis, osteomyelitis, intracranial abscess, and empyema were explicitly specified in some cases studies but not in others $[26,66]$. Our analysis therefore grouped all types of infection under a single category.

Third, our analysis did not stratify the data by age, sex, severity of injury, comorbidities, or other variables that may have influenced the results [88]. Neither did it account for the anatomic heterogeneity of $\mathrm{CP}$, as patients with unilateral, bilateral, and bifrontal craniectomies of various sizes were pooled together, even though bifrontal procedures may have significantly higher infection rates and increased risk for reoperation [89].

Fourth, the analysis treated each case of complication as an independent event. This may not be completely accurate, as in reality once a complication has accrued, it tends to predispose the patient for other complications [88].

Lastly, the analysis was susceptible to publication bias, as most of the data were derived from published articles, even though we integrated data from the literature with our direct surgical experience and performed separate analysis with and without the addition of our series. Overall results of the analysis of the two sets of data were largely in agreement, with the exception of post-CP hydrocephalus, as already discussed above.

Nonetheless, the idea behind this study was to try to shed light on the ongoing debate about whether or not TBI as primary pathology influences the rates of post-CP complications. The heterogeneity of the cohorts and data used for the analysis reflects the sacrifice of some of its validity in favor of generalizability, mirroring actual clinical practice, where the spectrum of CP patients, material, surgical techniques, and complications is wide and varied.

\section{Conclusion}

DC is an effective means to control ICP and mitigate the risk of herniation in patients with cerebral edema resulting from a wide range of conditions, and subsequent $\mathrm{CP}$ is crucial to restore cranial integrity and prevent SSFS [2]. However, while those procedures are technically straightforward, they place the patient at risk for many nontrivial complications, which can negatively impact outcome [86].

Our systematic review and meta-analysis investigated the difference in complication rates after $\mathrm{CP}$ following $\mathrm{DC}$, for TBI and all other indications considered together. Our results suggest that there is no significant difference in the overall complication rates between the two groups. However, when we analyzed specific complications separately, we found a significant increase in odds of autologous bone flap resorption and of infection in TBI patients. We hope that our findings will increase the awareness to these specific complications and promote the development of new strategies to decrease the risk of such complications, which may significantly compromise the already difficult pathway to recovery of trauma patients, even at advanced stages of healing.

Furthermore, as numbers of DC and CP continue to rise [90], it will become increasingly important to be aware of the actual risk of complications encountered by specific patient populations. Neurosurgeons, neurologists, and patients will need to know the risks of the procedure to be able to make informed decisions and develop new protocols to prevent those complications.

Supplementary Information The online version contains supplementary material available at https://doi.org/10.1007/s10143-021-01511-7.

Acknowledgements We would like to acknowledge all the researchers and surgeons that contributed to the studies and cases included in this review.

Code availability Not applicable.

Author contribution The study was conceived by DS and LM. Literature review, study selection, data collection, and statistical analysis were initially performed by DS and reviewed by LM. Institutional data collection was performed by GM. Analysis of the institutional data was performed by LM and GM. DS wrote the first draft of the manuscript that was further elaborated and revised by LM. All authors read and approved the final manuscript. 
Funding Open access funding provided by Università degli Studi di Pavia within the CRUI-CARE Agreement. LM was partially supported by a grant Ricerca Corrente (codice ric. 08016019) of the Fondazione IRCCS Policlinico S. Matteo.

Data availability All relevant data are contained in the manuscript and supplementary materials.

\section{Declarations}

Ethics approval For this type of purely retrospective study, formal consent is not required.

Consent to participate For the studies that had been published in the past, no consent to participate was necessary. For our local surgical series, all patients or their legal representative when the patient neurological status did not allow an informed decision signed an informed consent authorizing the use of anonymized clinical data for retrospective analysis and clinical research. Finally, all data and results are presented in aggregates and cannot be used to extract information on single patients.

\section{Consent for publication Not applicable.}

Competing interests The authors declare no competing interests.

Open Access This article is licensed under a Creative Commons Attribution 4.0 International License, which permits use, sharing, adaptation, distribution and reproduction in any medium or format, as long as you give appropriate credit to the original author(s) and the source, provide a link to the Creative Commons licence, and indicate if changes were made. The images or other third party material in this article are included in the article's Creative Commons licence, unless indicated otherwise in a credit line to the material. If material is not included in the article's Creative Commons licence and your intended use is not permitted by statutory regulation or exceeds the permitted use, you will need to obtain permission directly from the copyright holder. To view a copy of this licence, visit http://creativecommons.org/licenses/by/4.0/.

\section{References}

1. Hutchinson PJ, Kolias AG, Tajsic T, Adeleye A, Aklilu AT, Apriawan T, Bajamal AH, Barthélemy EJ, Devi BI, Bhat D, Bulters D, Chesnut R, Citerio G, Cooper DJ, Czosnyka M, Edem I, El-Ghandour NMF, Figaji A, Fountas KN, Gallagher C, Hawryluk GWJ, Iaccarino C, Joseph M, Khan T, Laeke T, Levchenko O, Liu B, Liu W, Maas A, Manley GT, Manson P, Mazzeo AT, Menon DK, Michael DB, Muehlschlegel S, Okonkwo DO, Park KB, Rosenfeld JV, Rosseau G, Rubiano AM, Shabani HK, Stocchetti N, Timmons SD, Timofeev I, Uff C, Ullman JS, Valadka A, Waran V, Wells A, Wilson MH, Servadei F (2019) Consensus statement from the International Consensus Meeting on the Role of Decompressive Craniectomy in the Management of Traumatic Brain Injury: consensus statement. Acta Neurochir (Wien) 161:1261-1274. https://doi.org/10. 1007/s00701-019-03936-y

2. Kolias AG, Kirkpatrick PJ, Hutchinson PJ (2013) Decompressive craniectomy: past, present and future. Nat Rev Neurol 9:405-415. https://doi.org/10.1038/nrneurol.2013.106

3. Acciarri N, Nicolini F, Martinoni M (2016) Cranioplasty: routine surgical procedure or risky operation? World J Surg Res 5:22-33
4. Sahoo NK, Tomar K, Thakral A, Rangan NM (2018) Complications of cranioplasty. J Craniofac Surg 29:1344-1348. https://doi.org/10.1097/SCS.0000000000004478

5. Sviri GE (2015) Massive cerebral swelling immediately after cranioplasty, a fatal and unpredictable complication: report of 4 cases. J Neurosurg 123:1188-1193. https://doi.org/10.3171/2014. 11.JNS141152

6. Ausman JI, Andrabi SM, Sarmast AH, Kirmani AR, Bhat AR (2017) Cranioplasty: indications, procedures, and outcome-an institutional experience. Surg Neurol Int 8:91. https://doi.org/10. 4103/sni.sni 4517

7. Killeen T, Fortunati M, Myanger E, Rüfenacht D, Ryskeldiyev N, Akshulakov S, Cesnulis E (2019) Symptomatic tension pneumocephalus following Palacos ${ }^{\circledR}$ cranioplasty in a shunted patient. Br J Neurosurg 33:215-216. https://doi.org/10.1080/ 02688697.2017 .1317718

8. Lang SS, Grady MS (2012) Surgical management of major skull defects and potential complications. In: Quinones-Hinojosa A (ed) Schmidek and Sweet Operative Neurosurgical Techniques: Indications, Methods, and Results, 6th edn. Saunders - Elsevier, Philadelphia, pp 1607-1610. https://doi.org/10.1016/B978-14160-6839-6.10140-6

9. Qiu S, You W, Wang H, Zhou X, Yang X (2019) Allergic epidural effusion following polyetheretherketone cranioplasty. J Craniofac Surg 30:E241-E243. https://doi.org/10.1097/SCS 0000000000005192

10. Honeybul S, Ho KM (2016) Cranioplasty: morbidity and failure. $\mathrm{Br}$ J Neurosurg 30:523-528. https://doi.org/10.1080/02688697.2016. 1187259

11. Rosseto RS, Giannetti AV, De Souza Filho LD, Faleiro RM (2015) Risk factors for graft infection after cranioplasty in patients with large hemicranial bony defects. World Neurosurg 84:431-437. https://doi.org/10.1016/j.wneu.2015.03.045

12. Walcott BP, Kwon CS, Sheth SA, Fehnel CR, Koffie RM, Asaad WF, Nahed BV, Coumans JV (2013) Predictors of cranioplasty complications in stroke and trauma patients. J Neurosurg 118: 757-762. https://doi.org/10.3171/2013.1.JNS121626

13. Kolias AG, Viaroli E, Rubiano AM, Adams H, Khan T, Gupta D, Adeleye A, Iaccarino C, Servadei F, Devi BI, Hutchinson PJ (2018) The current status of decompressive craniectomy in traumatic brain injury. Curr Trauma Reports 4:326-332. https://doi.org/10.1007/ s40719-018-0147-x

14. Khan AA, Banerjee A (2010) The role of prophylactic anticonvulsants in moderate to severe head injury. Int J Emerg Med 3:187191. https://doi.org/10.1007/s12245-010-0180-1

15. Riordan MA, Simpson VM, Hall WA (2016) Analysis of factors contributing to infections after cranioplasty: a single-institution retrospective chart review. World Neurosurg 87:207-213. https://doi. org/10.1016/j.wneu.2015.11.070

16. Schuss P, Vatter H, Oszvald A, Marquardt G, Imöhl L, Seifert V, Güresir E, Oszvald Á, Marquardt G, Imöhl L, Seifert V, Güresir E (2013) Bone flap resorption: risk factors for the development of a long-term complication following cranioplasty after decompressive craniectomy. J Neurotrauma 30:91-95. https://doi.org/10.1089/ neu.2012.2542

17. Moher D, Liberati A, Tetzlaff J, Altman DG, Altman D, Antes G, Atkins D, Barbour V, Barrowman N, Berlin JA, Clark J, Clarke M, Cook D, D'Amico R, Deeks JJ, Devereaux PJ, Dickersin K, Egger M, Ernst E, Gøtzsche PC, Grimshaw J, Guyatt G, Higgins J, Ioannidis JPA, Kleijnen J, Lang T, Magrini N, McNamee D, Moja L, Mulrow C, Napoli M, Oxman A, Pham B, Rennie D, Sampson M, Schulz KF, Shekelle PG, Tovey D, Tugwell P (2009) Preferred reporting items for systematic reviews and metaanalyses: the PRISMA statement. PLoS Med:6. https://doi.org/10. 1371/journal.pmed.1000097 
18. Wells G, Shea B, O'Connell D, Peterson J, Welch V, Losos M, Tugwell P (2006) The Newcastle-Ottawa Scale (NOS) for assessing the quality of nonrandomised studies in meta-analyses. Ottawa Heal Res Inst, In http://www.ohri.ca/programs/clinical_epidemiology/ oxford.asp. Accessed 30 May 2020

19. RevMan / Cochrane Training. https://training.cochrane.org/onlinelearning/core-software-cochrane-reviews/revman. Accessed 1 Jun 2020

20. Abode-Iyamah KO, Chiang HY, Winslow N, Park B, Zanaty M, Dlouhy BJ, Flouty OE, Rasmussen ZD, Herwaldt LA, Greenlee JD (2018) Risk factors for surgical site infections and assessment of vancomycin powder as a preventive measure in patients undergoing first-time cranioplasty. J Neurosurg 128:1241-1249. https://doi. org/10.3171/2016.12.JNS161967

21. Alkhaibary A, Alharbi A, Abbas M, Algarni A, Abdullah JM, Almadani WH, Khairy I, Alkhani A, Aloraidi A, Khairy S (2020) Predictors of surgical site infection in autologous cranioplasty: a retrospective analysis of subcutaneously preserved bone flaps in abdominal pockets. World Neurosurg 133:e627-e632. https://doi. org/10.1016/j.wneu.2019.09.120

22. Archavlis E, Carvi Y, Nievas M (2012) The impact of timing of cranioplasty in patients with large cranial defects after decompressive hemicraniectomy. Acta Neurochir (Wien) 154:10551062. https://doi.org/10.1007/s00701-012-1333-1

23. Beauchamp KM, Kashuk J, Moore EE, Bolles G, Rabb C, Seinfeld J, Szentirmai O, Sauaia A (2010) Cranioplasty after postinjury decompressive craniectomy: is timing of the essence? J Trauma 69: 270-274. https://doi.org/10.1097/TA.0b013e3181e491c2

24. Bobinski L, Koskinen L-OD, Lindvall P (2013) Complications following cranioplasty using autologous bone or polymethylmethacrylate - retrospective experience from a single center. Clin Neurol Neurosurg 115:1788-1791. https://doi.org/10. 1016/j.clineuro.2013.04.013

25. Borger V, Schuss P, Kinfe TMTMTM, Vatter H, Güresir E (2016) Decompressive craniectomy for stroke: early cranioplasty is a predictor for postoperative complications. World Neurosurg 92:83-88. https://doi.org/10.1016/j.wneu.2016.04.113

26. Von Der Brelie C, Stojanovski I, Meier U, Lemcke J (2015) Open traumatic brain injury is a strong predictor for aseptic bone necrosis after cranioplasty surgery: a retrospective analysis of 219 patients. $\mathrm{J}$ Neurol Surgery, Part A Cent Eur Neurosurg 77:19-24. https://doi. org $/ 10.1055 / \mathrm{s}-0035-1558410$

27. Brommeland T, Rydning PN, Pripp AH, Helseth E (2015) Cranioplasty complications and risk factors associated with bone flap resorption. Scand J Trauma Resusc Emerg Med:23. https://doi. org/10.1186/s13049-015-0155-6

28. Chang V, Hartzfeld P, Langlois M, Mahmood A, Seyfried D (2010) Outcomes of cranial repair after craniectomy: Clinical article. J Neurosurg 112:1120-1124. https://doi.org/10.3171/2009.6. JNS09133

29. Chaturvedi J, Botta R, Prabhuraj AR, Shukla D, Bhat DI, Indira Devi B (2016) Complications of cranioplasty after decompressive craniectomy for traumatic brain injury. Br J Neurosurg 30:264-268. https://doi.org/10.3109/02688697.2015.1054356

30. Chen S-T, Chang C-J, Su W-C, Chang L-W, Chu I-H, Lin M-S (2015) 3-D titanium mesh reconstruction of defective skull after frontal craniectomy in traumatic brain injury. Injury 46:80-85. https://doi.org/10.1016/j.injury.2014.09.019

31. Cheng C-HCH, Lee HCH-C, Chen CCC-C, Cho DYD-Y, Lin HLH-L (2014) Cryopreservation versus subcutaneous preservation of autologous bone flaps for cranioplasty: comparison of the surgical site infection and bone resorption rates. Clin Neurol Neurosurg 124:85-89. https://doi.org/10.1016/j.clineuro.2014.06.029

32. Cheng YK, Weng HH, Yang JT, Lee MH, Wang TC, Chang CN (2008) Factors affecting graft infection after cranioplasty. J Clin
Neurosci 15:1115-1119. https://doi.org/10.1016/j.jocn.2007.09. 022

33. Daou B, Zanaty M, Chalouhi N, Dalyai R, Jabbour P, Yang S, Rosenwasser RH, Tjoumakaris S (2016) Low incidence of bone flap resorption after native bone cranioplasty in adults. World Neurosurg 92:89-94. https://doi.org/10.1016/j.wneu.2016.04.115

34. Dünisch P, Walter J, Sakr Y, Kalff R, Waschke A, Ewald C (2013) Risk factors of aseptic bone resorption: a study after autologous bone flap reinsertion due to decompressive craniotomy - Clinical article. J Neurosurg 118:1141-1147. https://doi.org/10.3171/2013. 1.JNS12860

35. Goedemans T, Verbaan D, van der Veer O, Bot M, Post R, Hoogmoed J, Lequin MB, Buis DR, Vandertop WP, Coert BA, van den Munckhof P (2020) Complications in cranioplasty after decompressive craniectomy: timing of the intervention. J Neurol 267:1312-1320. https://doi.org/10.1007/s00415-020-09695-6

36. Güresir E, Vatter H, Schuss P, Oszvald Á, Raabe A, Seifert V, Beck J (2011) Rapid closure technique in decompressive craniectomy. Clinical article. J Neurosurg 114:954-960. https://doi.org/10. 3171/2009.12.JNS091065

37. Huang Y-HH, Lee T-CC, Chen W-FF, Wang Y-MM (2011) Safety of the nonabsorbable dural substitute in decompressive craniectomy for severe traumatic brain injury. J Trauma 71:533-537. https://doi. org/10.1097/TA.0b013e318203208a

38. Im SH, Jang DK, Han YM, Kim JT, Chung DS, Park YS (2012) Long-term incidence and predicting factors of cranioplasty infection after decompressive craniectomy. J Korean Neurosurg Soc 52: 396-403. https://doi.org/10.3340/jkns.2012.52.4.396

39. Inamasu J, Kuramae T, Nakatsukasa M (2010) Does difference in the storage method of bone flaps after decompressive craniectomy affect the incidence of surgical site infection after cranioplasty? Comparison between subcutaneous pocket and cryopreservation. J Trauma 68:183-187. https://doi.org/10.1097/TA. 0b013e3181c45384

40. Iwama T, Yamada J, Imai S, Shinoda J, Funakoshi T, Sakai N, Sekhar LN, Stimac D, Sindou MP, Haines SJ, Kawase T (2003) The use of frozen autogenous bone flaps in delayed cranioplasty revisited. Neurosurgery 52:591-596. https://doi.org/10.1227/01. NEU.0000047891.86938.46

41. Jin SW, Kim SD, Ha SK, Lim DJ, Lee H, You HJ (2018) Analysis of the factors affecting surgical site infection and bone flap resorption after cranioplasty with autologous cryopreserved bone: the importance of temporalis muscle preservation. Turk Neurosurg 28: 882-888. https://doi.org/10.5137/1019-5149.JTN.21333-17.2

42. Kim JH, Hwang SY, Kwon TH, Chong K, Yoon WK, Kim JH (2019) Defining "early" cranioplasty to achieve lower complication rates of bone flap failure: resorption and infection. Acta Neurochir (Wien) 161:25-31. https://doi.org/10.1007/s00701-018-3749-8

43. Klinger DR, Madden C, Beshay J, White J, Gambrell K, Rickert K (2014) Autologous and acrylic cranioplasty: a review of 10 years and 258 cases. World Neurosurg. 82:E525-E530

44. Krause-Titz UR, Warneke N, Freitag-Wolf S, Barth H, Mehdorn HM (2016) Factors influencing the outcome (GOS) in reconstructive cranioplasty. Neurosurg Rev 39:133-139. https://doi.org/10. 1007/s10143-015-0678-3

45. Kriegel RJ, Schaller C, Clusmann H (2007) Cranioplasty for large skull defects with PMMA (polymethylmethacrylate) or tutoplast ${ }^{\circledR}$ processed autogenic bone grafts. Zentralbl Neurochir 68:182-189. https://doi.org/10.1055/s-2007-985857

46. Lee JW, Kim JH, Kang HI, Moon BG, Lee SJ, Kim JS (2011) Epidural fluid collection after cranioplasty: fate and predictive factors. J Korean Neurosurg Soc 50:231-234. https://doi.org/10.3340/ jkns.2011.50.3.231

47. Lee L, Ker J, Quah BL, Chou N, Choy D, Yeo TT (2013) A retrospective analysis and review of an institution's experience with 
the complications of cranioplasty. Br J Neurosurg 27:629-635. https://doi.org/10.3109/02688697.2013.815313

48. Matsuno A, Tanaka H, Iwamuro H, Takanashi S, Miyawaki S, Nakashima M, Nakaguchi H, Nagashima T (2006) Analyses of the factors influencing bone graft infection after delayed cranioplasty. Acta Neurochir (Wien) 148:535-540. https://doi.org/ 10.1007/s00701-006-0740-6

49. Moreira-Gonzalez A, Jackson IT, Miyawaki T, Barakat K, DiNick V (2003) Clinical outcome in cranioplasty: critical review in longterm follow-up. J Craniofac Surg. 14:144-153. https://doi.org/10. 1097/00001665-200303000-00003

50. Morton RP, Abecassis IJ, Hanson JF, Barber JK, Chen M, Kelly CM, Nerva JD, Emerson SN, Ene CI, Levitt MR, Chowdhary MM, Ko AL, Chesnut RM (2018) Timing of cranioplasty: a 10.75-year single-center analysis of 754 patients. J Neurosurg 128:1648-1652. https://doi.org/10.3171/2016.11.JNS161917

51. Mukherjee S, Thakur B, Haq I, Hettige S, Martin AJAJ (2014) Complications of titanium cranioplasty - a retrospective analysis of 174 patients. Acta Neurochir (Wien) 156:989-998. https://doi. org/10.1007/s00701-014-2024-x

52. Nagayama K, Yoshikawa G, Somekawa K, Kohno M, Segawa H, Sano K, Shiokawa Y, Saito I (2002) Cranioplasty using the patient's autogenous bone preserved by freezing - an examination of post-operative infection rates. No Shinkei Geka 30:165-169

53. Oladunjoye AO, Schrot RJ, Zwienenberg-Lee M, Muizelaar JP, Shahlaie K (2013) Decompressive craniectomy using gelatin film and future bone flap replacement. J Neurosurg 118:776-782. https://doi.org/10.3171/2013.1.JNS121475

54. Paredes I, Castaño-León AM, Munarriz PM, Martínez-Perez R, Cepeda S, Sanz R, Alén JF, Lagares A (2015) Cranioplasty after decompressive craniectomy. A prospective series analyzing complications and clinical improvement. Neurocirugia 26:115-125. https://doi.org/10.1016/j.neucir.2014.10.001

55. Piedra MP, Ragel BT, Dogan A, Coppa ND, Delashaw JB (2013) Timing of cranioplasty after decompressive craniectomy for ischemic or hemorrhagic stroke: Clinical article. J Neurosurg 118:109114. https://doi.org/10.3171/2012.10.JNS121037

56. Pierson M, Birinyi PV, Bhimireddy S, Coppens JR (2016) Analysis of decompressive craniectomies with subsequent cranioplasties in the presence of collagen matrix dural substitute and polytetrafluoroethylene as an adhesion preventative material. World Neurosurg 86:153-160. https://doi.org/10.1016/j.wneu. 2015.09.078

57. Posti JP, Yli-Olli M, Heiskanen L, Aitasalo KMJ, Rinne J, Vuorinen V, Serlo W, Tenovuo O, Vallittu PK, Piitulainen JM (2018) Cranioplasty after severe traumatic brain injury: effects of trauma and patient recovery on cranioplasty outcome. Front Neurol 9:223-229. https://doi.org/10.3389/fneur.2018.00223

58. Quah BL, Low HL, Wilson MH, Bimpis A, Nga VDW, Lwin S, Zainuddin NH, Wahab NA, Salek MAA (2016) Is there an optimal time for performing cranioplasties? Results from a prospective multinational study. World Neurosurg 94:13-17. https://doi.org/10. 1016/j.wneu.2016.06.081

59. Rashidi A, Sandalcioglu IE, Luchtmann M (2020) Aseptic boneflap resorption after cranioplasty - incidence and risk factors. PLoS One 15:e0228009. https://doi.org/10.1371/journal.pone.0228009

60. Roberts SAG, Toman E, Belli A, Midwinter MJ (2016) Decompressive craniectomy and cranioplasty: experience and outcomes in deployed UK military personnel. Br J Neurosurg 30:529535. https://doi.org/10.1080/02688697.2016.1208807

61. Schoekler B, Trummer M (2014) Prediction parameters of bone flap resorption following cranioplasty with autologous bone. Clin Neurol Neurosurg 120:64-67. https://doi.org/10.1016/j.clineuro. 2014.02.014

62. Schuss P, Vatter H, Marquardt G, Imöhl L, Ulrich CT, Seifert V, Güresir E (2012) Cranioplasty after decompressive craniectomy: the effect of timing on postoperative complications. J Neurotrauma 29:1090-1095. https://doi.org/10.1089/neu.2011. 2176

63. Shibahashi K, Hoda H, Takasu Y, Hanakawa K, Ide T, Hamabe Y (2017) Cranioplasty outcomes and analysis of the factors influencing surgical site infection: a retrospective review of more than 10 years of institutional experience. World Neurosurg 101:20-25. https://doi.org/10.1016/j.wneu.2017.01.106

64. Shih F-Y, Lin C-C, Wang H-C, Ho J-T, Lin C-H, Lu Y-T, Chen WF, Tsai M-H (2019) Risk factors for seizures after cranioplasty. Seizure 66:15-21. https://doi.org/10.1016/j.seizure.2018.12.016

65. Shimizu S, Morikawa A, Kuga Y, Mouri G, Murata T (2002) Cranioplasty using autogenous bone cryopreserved with dimethylsulfoxide (DMSO). No Shinkei Geka 30:479-485

66. Sobani ZA, Shamim MS, Qadeer M, Murtaza S, Bari M, Sobani Z, Zafar S, Bilal N, Enam S (2011) Cranioplasty after decompressive craniectomy: an institutional audit and analysis of factors related to complications. Surg Neurol Int 2:123. https://doi.org/10.4103/ 2152-7806.85055

67. Stephens FL, Mossop CM, Bell RS, Tigno T, Rosner MK, Kumar A, Moores LE, Armonda RA (2010) Cranioplasty complications following wartime decompressive craniectomy. Neurosurg Focus 28:E3. https://doi.org/10.3171/2010.2.FOCUS1026

68. Sundseth J, Sundseth A, Berg-Johnsen J, Sorteberg W, Lindegaard K-F (2014) Cranioplasty with autologous cryopreserved bone after decompressive craniectomy. Complications and risk factors for developing surgical site infection. Acta Neurochir (Wien) 156:805811. https://doi.org/10.1007/s00701-013-1992-6

69. Tantawi D, Armonda R, Valerio I, Kumar AR (2012) Management of decompressive craniectomy defects: modern military treatment strategies. J Craniofac Surg 23:2042-2045. https://doi.org/10.1097/ SCS.0b013e318258ba36

70. Tsang ACO, Hui VKH, Lui WM, Leung GKK (2015) Complications of post-craniectomy cranioplasty: risk factor analysis and implications for treatment planning. J Clin Neurosci 22: 834-837. https://doi.org/10.1016/j.jocn.2014.11.021

71. Wachter D, Reineke K, Behm T, Rohde V (2013) Cranioplasty after decompressive hemicraniectomy: underestimated surgeryassociated complications? Clin Neurol Neurosurg 115:12931297. https://doi.org/10.1016/j.clineuro.2012.12.002

72. Yeap MC, Chen CC, Liu ZH, Hsieh PC, Lee CC, Liu YT, Yi-Chou Wang A, Huang YC, Wei KC, Wu CT, Tu PH (2018) Postcranioplasty seizures following decompressive craniectomy and seizure prophylaxis: a retrospective analysis at a single institution. J Neurosurg 131:936-940. https://doi.org/10.3171/2018.4. JNS172519

73. Honeybul S (2010) Complications of decompressive craniectomy for head injury. J Clin Neurosci 17:430-435. https://doi.org/10. 1016/j.jocn.2009.09.007

74. Honeybul S, Ho KM (2012) How "successful" is calvarial reconstruction using frozen autologous bone? Plast Reconstr Surg 130: 1110-1117. https://doi.org/10.1097/PRS.0b013e318267d4de

75. Huang Y-H, Yang T-M, Lee T-C, Chen W-F, Yang K-Y (2013) Acute autologous bone flap infection after cranioplasty for postinjury decompressive craniectomy. Injury 44:44-47. https:// doi.org/10.1016/j.injury.2011.11.005

76. Durham SR, McComb JG, Levy ML, Cohen AR, Menezes AH, Sutton LN (2003) Correction of large $(>25 \mathrm{~cm} 2)$ cranial defects with "reinforced" hydroxyapatite cement: technique and complications. Neurosurgery 52:842-845. https://doi.org/10.1227/01.NEU. $0000054220.01290 .8 \mathrm{E}$

77. Gilardino MS, Cabiling DS, Bartlett SP (2009) Long-term followup experience with carbonated calcium phosphate cement (norian) for cranioplasty in children and adults. Plast Reconstr Surg 123: 983-994. https://doi.org/10.1097/PRS.0b013e318199f6ad 
78. Poetker DM, Pytynia KB, Meyer A, Wackym PA (2004) Complication rate of transtemporal hydroxyapatite cement cranioplasties: a case series review of 76 cranioplasties. Otol Neurotol. 25:604-609. https://doi.org/10.1097/00129492200407000-00031

79. Godil SS, Shamim MS, Enam SA, Qidwai U, Qadeer M, Sobani ZA (2011) Cranial reconstruction after decompressive craniectomy: prediction of complications using fuzzy logic. J Craniofac Surg 22: 1307-1311. https://doi.org/10.1097/SCS.0b013e31821c6d37

80. Honeybul S, Ho KM (2011) Long-term complications of decompressive craniectomy for head injury. J Neurotrauma 28:929-935. https://doi.org/10.1089/neu.2010.1612

81. Grant GA, Jolley M, Ellenbogen RG, Roberts TS, Gruss JR, Loeser JD (2004) Failure of autologous bone-assisted cranioplasty following decompressive craniectomy in children and adolescents. J Neurosurg 100:163-168. https://doi.org/10.3171/ped.2004.100.2. 0163

82. Stiver SI (2009) Complications of decompressive craniectomy for traumatic brain injury. Neurosurg. Focus 26:E7. https://doi.org/10. 3171/2009.4.FOCUS0965

83. Bowers CA, Riva-Cambrin JAY, Hertzler DA, Walker ML (2013) Risk factors and rates of bone flap resorption in pediatric patients after decompressive craniectomy for traumatic brain injury. Clinical article. J Neurosurg Pediatr 11:526-532. https://doi.org/10.3171/ 2013.1.PEDS12483

84. Thavarajah D, De Lacy P, Hussien A, Sugar A (2012) The minimum time for cranioplasty insertion from craniectomy is six months to reduce risk of infection-a case series of 82 patients. Br J Neurosurg 26:78-80. https://doi.org/10.3109/02688697.2011. 603850
85. Al-Qattan H, Gernsback JE, Nugent AG, Lyapichev KA, Komotar RJ, Chim H (2017) Heterotopic intracranial skin presenting as chronic draining sinus after remote craniotomy. World Neurosurg 98(882):e9-e14. https://doi.org/10.1016/j.wneu.2016.10.094

86. Kurland DB, Khaladj-Ghom A, Stokum JAJA, Carusillo B, Karimy JK, Gerzanich V, Sahuquillo J, Simard JM (2015) Complications associated with decompressive craniectomy: a systematic review. Neurocrit Care 23:292-304. https://doi.org/10.1007/s12028-0150144-7

87. Yadla S, Campbell PG, Chitale R, Maltenfort MG, Jabbour P, Sharan AD (2011) Effect of early surgery, material, and method of flap preservation on cranioplasty infections: a systematic review. Neurosurgery 68:1124-1130

88. Spencer R, Manivannan S, Sharouf F, Bhatti MI, Zaben M (2019) Risk factors for the development of seizures after cranioplasty in patients that sustained traumatic brain injury: a systematic review. Seizure 69:11-16. https://doi.org/10.1016/j.seizure.2019.03.014

89. Gooch MR, Gin GE, Kenning TJ, German JW, Reid Gooch M, Gin GE, Kenning TJ, German JW (2009) Complications of cranioplasty following decompressive craniectomy: analysis of 62 cases. Neurosurg Focus 26:E9. https://doi.org/10.3171/2009.3. FOCUS0962

90. Walcott BP, Kuklina EV, Nahed BV, George MG, Kahle KT, Simard JM, Asaad WF, Coumans JVCE (2011) Craniectomy for malignant cerebral infarction: prevalence and outcomes in US hospitals. PLoS One 6:e29193. https://doi.org/10.1371/journal.pone. 0029193

Publisher's note Springer Nature remains neutral with regard to jurisdictional claims in published maps and institutional affiliations. 